\title{
Recent Trends in the Application of Chromatographic Techniques in the Analysis of Luteolin and Its Derivatives
}

\author{
Aleksandra Maria Juszczak $^{1}\left(\mathbb{D}\right.$, Marijana Zovko-Končić $^{2}$ and Michał Tomczyk ${ }^{1, * \mathbb{C}}$ \\ 1 Department of Pharmacognosy, Faculty of Pharmacy, Medical University of Białystok, ul. Mickiewicza 2a, \\ 15-230 Białystok, Poland; aleksandra.juszczak@umb.edu.pl \\ 2 Department of Pharmacognosy, University of Zagreb, Faculty of Pharmacy and Biochemistry, A. Kovačića 1, \\ 10000 Zagreb, Croatia; mzovko@pharma.hr \\ * Correspondence: michal.tomczyk@umb.edu.pl; Tel.: +48-85-748-5694
}

Received: 1 October 2019; Accepted: 8 November 2019; Published: 12 November 2019

check for updates

\begin{abstract}
Luteolin is a flavonoid often found in various medicinal plants that exhibits multiple biological effects such as antioxidant, anti-inflammatory and immunomodulatory activity. Commercially available medicinal plants and their preparations containing luteolin are often used in the treatment of hypertension, inflammatory diseases, and even cancer. However, to establish the quality of such preparations, appropriate analytical methods should be used. Therefore, the present paper provides the first comprehensive review of the current analytical methods that were developed and validated for the quantitative determination of luteolin and its $C$ - and $\mathrm{O}$-derivatives including orientin, isoorientin, luteolin 7-O-glucoside and others. It provides a systematic overview of chromatographic analytical techniques including thin layer chromatography (TLC), high performance thin layer chromatography (HPTLC), liquid chromatography (LC), high performance liquid chromatography (HPLC), gas chromatography (GC) and counter-current chromatography (CCC), as well as the conditions used in the determination of luteolin and its derivatives in plant material.
\end{abstract}

Keywords: luteolin; hyphenated techniques; chromatography

\section{Introduction}

Luteolin (Figure 1) is a yellow dye commonly found in fresh plants. It is a flavonoid of the flavone type that is distributed widely throughout the plant kingdom. Similar to other derivatives of 2-phenylbenzo- $\gamma$-pyrone, its basic skeleton has a characteristic $C_{6}-C_{3}-C_{6}$ system, containing two benzene rings and a bridge with a C2-C3 double carbon bond and an attached oxygen atom [1-4]. Structure-activity studies have demonstrated that the pharmacological effects of luteolin and other flavonoids are strongly related to the presence of hydroxyl groups at the $\mathrm{C} 5, \mathrm{C} 7, \mathrm{C} 3$ ' and $\mathrm{C} 4$ ' carbons as well as to the presence of the double bond in the $\mathrm{C} 2-\mathrm{C} 3$ position $[3,5]$. The presence of the -OH group at position $\mathrm{C}^{\prime}$ ' distinguishes luteolin from apigenin, and the lack of this group at the C3 carbon is an element that places luteolin in the flavone group [6]. 
<smiles>O=c1cc(-c2ccc(O)c(O)c2)oc2cc(O)cc(O)c12</smiles>

Figure 1. Chemical structure of luteolin.

Luteolin exhibits multiple biological effects such as antioxidant, anti-inflammatory and immunomodulatory activity. Plants rich in luteolin are often used in traditional medicine for treatment of various diseases such as hypertension, inflammatory disorders, and even cancer [7].

Because luteolin bears four hydroxyl groups (at the C5, C7, C3' and C4' positions), many derivatives of luteolin can be created. Various types of functional groups and/or sugar molecules can be attached to those positions, creating a huge number of different but structurally similar molecules. The most common are methyl derivatives, as well as $\mathrm{C}$ - and -O-glycosides [8,9].

Orientin, an 8-C-glucoside derivative of luteolin, displays an array of health-related biological properties, such as antioxidant, anti-ageing, antiviral, antibacterial, anti-inflammatory, vasodilatation, cardioprotective, radiation protective, neuroprotective, antidepressant-like, anti-adipogenesis, and antinociceptive effects. It may be found in different medicinal plants such as Ocimum sanctum (holy basil), Phyllostachys nigra (bamboo leaves), Passiflora sp. (passion flower), Linum usitatissimum (flax), Euterpe oleracea (Acai palm) and many others [10]. Another luteolin derivative, isoorientin (luteolin-6-C-glucoside) acts as an antioxidant, photoprotective [11], skin lightening [12], hepatoprotective [13] and anti-inflammatory agent [14]. O-glucosides of luteolin also display biological activities. For example, luteolin 7-O-glucoside alleviates skin lesions in murine models of atopic dermatitis [15] and protects cells against apoptosis induced by hypoxia/reoxygenation [16].

An increasing number of herbal preparations on the market contain luteolin and its derivatives, either as single-ingredient products or in mixtures with other phytochemicals, e.g., in form of medicinal plants extracts. To establish the quality of such products, it is important to use appropriate analytical methods. However, there is a lack of quality reviews of the available methods for quantification of luteolin derivatives, and the information on their comparison is lacking. Therefore, the aim of this article is to systematize knowledge and information in the field of chromatographic analytical techniques used for quantification of luteolin and its derivatives. The presented review is the first description of this type and provides a systematic overview of chromatographic analytical techniques including thin layer chromatography (TLC), high performance thin layer chromatography (HPTLC), liquid chromatography (LC), high performance liquid chromatography (HPLC), gas chromatography (GC) and counter-current chromatography (CCC), as well as the conditions used to assess luteolin and its derivatives.

\section{Chromatographic Techniques for the Analysis of Luteolin Derivatives}

Chromatography occupies a leading position among other instrumental methods in the analysis of chemical compounds. As a physicochemical method of separation and analysis of mixtures of chemical compounds, it allows detection and identification as well as quantitative determination of the test substance with high accuracy. The coupling of chromatography with other methods of analysis contributes to a more accurate detection and expansion of analytical capabilities, especially for complex mixtures of organic compounds $[2,17,18]$. 
Chromatographic techniques are based on the interaction of the mixture components with the mobile and stationary phases of the chromatographic system. This results in the division of the mixture components between the two phases. In addition, the interaction of the mobile and stationary phases is also important in the separation process $[17,18]$. According to the aggregation state of the mobile phase, chromatographic techniques are divided into gas, liquid and supercritical chromatography. Another criterion for classification of chromatographic techniques is the type of stationary phase. If the stationary phase is a liquid, the chromatography technique is referred to as partition chromatography. In the case of a solid, the technique is referred to as adsorption chromatography. Another example of the classification of chromatographic methods is their division depending on the chromatographic processing method. This classification allows distinguishing column chromatography and planar techniques, which include TLC and paper chromatography $[17,19]$. Many different chromatographic techniques are used in the analysis of luteolin derivatives. These include TLC, HPTLC, LC, HPLC, GC and CCC [19].

\subsection{Thin Layer Chromatography in the Analysis of Luteolin Derivatives}

Thin layer chromatography (TLC) is a rather simple but relatively popular method used in the analysis of flavonoids since 1960 [20]. It is a variation of LC that is carried out on a plane and is therefore referred to as planar chromatography. Despite the dynamic development of other chromatography techniques, TLC has not lost its importance in phytochemical analysis [18]. Its values are still recognized as is the basic tool in the qualitative analysis of natural products, and as such, it is still recommended by most modern Pharmacopoeias. In addition, the TLC technique is currently being improved, and the scope of its application is widening, while the results are becoming comparable to those obtained by GC or HPLC [20-22]. The stages of chromatographic analysis consist of placing the sample on the stationary phase and developing the chromatogram, followed by its visualization. In the final step, qualitative and/or quantitative determinations of the tested components are made [18,22,23]. General guidelines for flavonoid separation on TLC plates are presented in Table 1.

Table 1. Recommended combinations of solvents / adsorbents for identification. of different flavonoid types by thin layer chromatography (TLC).

\begin{tabular}{|c|c|c|c|}
\hline \multirow{2}{*}{ Flavonoid Type } & \multicolumn{3}{|c|}{ Adsorbent Type/Mobile Phase } \\
\hline & Cellulose & Polyamide & Silica gel \\
\hline $\begin{array}{l}\text { Polar flavonoid } \\
\text { aglycones, e.g., flavones }\end{array}$ & $\begin{array}{c}\mathrm{BuOH}: \mathrm{AcOH}: \mathrm{H}_{2} \mathrm{O}(3: 1: 1 \\
v / v / v)^{\mathrm{a}} \\
\mathrm{CHCl}_{3}: \mathrm{AcOH}: \mathrm{H}_{2} \mathrm{O} \\
(30: 15: 2 v / v / v)^{\mathrm{b}}\end{array}$ & $\begin{array}{c}\text { MeOH:AcOH: } \mathrm{H}_{2} \mathrm{O} \\
(18: 1: 1 v / v / v)\end{array}$ & To:Py:FA (36:9:5 v/v/v) \\
\hline $\begin{array}{l}\text { Non-polar flavonoid } \\
\text { aglycones, e.g., } \\
\text { methylated flavones }\end{array}$ & $10-30 \% \mathrm{AcOH}$ & - & $\begin{array}{c}\mathrm{CHCl}_{3}: \mathrm{MEOH}(15: 1 \text { to } \\
3: 1 \mathrm{v} / v)\end{array}$ \\
\hline Flavonoid glycosides & $\begin{array}{c}\text { BuOH:AcOH: } \mathrm{H}_{2} \mathrm{O}(3: 1: 1 \\
v / v / v)^{\mathrm{a}} \\
\text { BuOH:AcOH: } \mathrm{H}_{2} \mathrm{O}(4: 1: 5 \\
v / v / v)^{\mathrm{a}}\end{array}$ & $\begin{array}{c}\mathrm{H}_{2} \mathrm{O}: \mathrm{MeOH} \text { :MEK:methyl } \\
\text { acetylacetone }(13: 3: 3: 1 \\
v / v / v / v)\end{array}$ & $\begin{array}{c}\text { EtOAc:Py: } \mathrm{H}_{2} \mathrm{O}: \mathrm{MeOH} \\
\text { (80:20:10:5 v/v/v/v) } \\
\text { (especially flavone } \\
\text { C-glycosides) }\end{array}$ \\
\hline
\end{tabular}

Abbreviations: $\mathrm{AcOH}$, acetic acid; $\mathrm{BuOH}$, butanol; $\mathrm{CHCl}$, chloroform; EtOAc, ethyl acetate; $\mathrm{FA}$, formic acid; $\mathrm{MeOH}$, methanol; MEK, methyl ethyl ketone; Py, pyridine; To, toluene. ${ }^{a}$ The mobile phase is thoroughly mixed in the separating funnel and the upper phase is used. ${ }^{\mathrm{b}}$ The mobile phase is thoroughly mixed in the separating funnel and the excess water is discarded. 
The main advantage of TLC is that it is a relatively simple and inexpensive technique that allows for rapid qualitative and quantitative analysis of the tested compounds. Samples analyzed with this method usually do not require pre-treatment, such as purification or concentration. In addition, several dozens of samples can be analyzed simultaneously on one plate. A large amount of the diluted sample can be applied to the stationary phase because the solvent evaporates during the application to the plate. Furthermore, due to the evaporation of the solvent phase after the development of the chromatogram, the detection method does not depend on the type of mobile phase used for separation. In TLC, it is possible not only to compare the analyzed components with the standards, but also to differentiate between substances bearing specific functional groups using the appropriate reagents for detection [18].

Thin layer chromatography and column chromatography (CC) are interchangeable techniques that may be combined, which significantly reduces costs and analysis time [22]. To achieve this, the same adsorbents are ideally used for both TLC and CC [18,22,24]. Nevertheless, other solvent systems may also be used. Elution in CC may be carried out in one mobile phase, or its composition may be changed during chromatography (mobile phase gradient), thereby increasing the elution force. In this case, the TLC mobile phase should be changed accordingly [18,24].

The type and quality of the stationary phase greatly affect the separation of mixture components. Thus, the selection of an appropriate adsorbent is very important. However, most TLC analyses are carried out in a normal phase system where hydrophilic (polar) adsorbents are used [18,23,24]. Reversed phase systems with lipophilic (non-polar) stationary phases are common and have little significance in the analysis of flavonoids [20]. Currently, the most commonly used stationary phase for the analysis of flavonoids is silica gel [20]. However, the use polyamide coated chromatography plates, in both normal and reversed phase systems, is not uncommon [24].

Detection of flavonoids on TLC plates is most often conducted under ultraviolet (UV) light at wavelengths of 254 or $366 \mathrm{~nm}$. Luteolin derivatives also display fluorescence, which can be enhanced using the appropriate derivatization reagents, e.g., with the so-called NP/PEG reagent. The most frequent procedure consists of spraying the plate with $1 \%$ methanolic diphenylboric acid- $\beta$-ethylamino ester (natural product reagents, NP), followed by $5 \%$ ethanolic polyethylene glycol 4000 (PEG) solution [20]. A densitometer may also be used for the qualitative analysis of the substance. The analysis is performed by comparing the retardation factor (Rf) and absorption spectrum of the test substance and the standard. Analytes can also be identified by extracting the separated substances from the plate. Then, the analysis is carried out using Fourier transform infrared mass spectrometry (MS), UV spectrometry, Raman spectrometry or other techniques [23]. Even though TLC separation of luteolin derivatives (Table 2) can be performed in different types of stationary phases such as a polyamide phase [26,28], it is most frequently performed on silica gel plates that are often coated with a fluorescent indicator [27,29] $\left(\mathrm{F}_{254}\right.$ plates) for preliminary detection. Such an approach has been used in case of analysis of luteolin 3'-O-glucoside, luteolin 6,8-C-dihexoside and luteolin 7-O-rutinoside in Phlomis sp. [27]. However, subsequent analysis with NP/PEG is the standard procedure for TLC analysis of luteolin [26-29], and it is almost always performed regardless of additional types of detection such as detection of flavonoids in Ligustrum vulgare with aniline phthalate [26]. Typically, the mobile phase for luteolin derivative separation consists of a mixture of aprotic organic solvents such as ethyl acetate (EtOAc) $[26,28]$ or acetone (Ace) [29] and $\mathrm{H}_{2} \mathrm{O}$ with a significant amount of formic (FA) and/or acetic acid $(\mathrm{AcOH})$ to avoid tailing of the separated zones [26-29]. 
Thin layer chromatography is often used as a complementary method to other chromatographic techniques. For example, analysis of the butanol $(\mathrm{BuOH})$ fraction of the methanol $(\mathrm{MeOH})$ extract from the leaves of the common privet (Ligustrum vulgare) conducted by Mučaji et al. [26] allowed isolation of two luteolin derivatives from the plant. TLC was carried out, among other techniques, on polyamide plates, and the optimal mobile phase was found. TLC was used for analysis of individual fractions obtained in column chromatography with or without acid hydrolysis of compounds. MS detection and nuclear magnetic resonance (NMR) spectra were also used [26]. Furthermore, TLC was used together with high performance liquid chromatography combined with mass spectrometry and pulsed amperometric detection (HPLC-PAD-MS) for analysis of luteolin and other phenolic compounds in Leontopodium alpinum. In addition to NP/PEG, UV, infrared (IR) and NMR analyses were used for identification of these compounds [29].

Compared to other chromatographic techniques, HPTLC results in reduced time and costs of analysis and provides much greater efficiency of separation. It is suitable even for the analysis of crude extracts similar to TLC, and a relatively small amount of solvent is used to analyze several samples, making this method environmentally friendly [60]. In the analysis of luteolin derivatives (Table 3), HPTLC silica gel 60 is almost exclusively used as the stationary phase $[45,47,49,51]$, while $\mathrm{HPTLC} \mathrm{NH}_{2}$ plates are rarely used, e.g., for separation of flavonoids in some Lamiaceae species such as Mentha piperita [53] and Thymus sp. [55]. In addition to NP/PEG (e.g., [52,57]), other detection systems may be employed for visualization of luteolin derivatives, such as bis-diazotized sulfanilamide [53] or aqueous solutions of $\mathrm{Al}^{3+}$ ions for flavonoids in M. piperita [53], honey [49] or Thymus sp. [55]. Similar to TLC, mixtures of organic solvents, $\mathrm{H}_{2} \mathrm{O}$, and FA are most often used as the mobile phase $[44,45,47-49,51,52]$.

High performance thin layer chromatography may also be used as a complimentary method to other chromatographic techniques. Chelyn et al. [44] used the HPTLC technique in the analysis of the ethanol (EtOH) extract of Clinacanthus nutans leaves. The analysis revealed the presence of, among others, luteolin 8-C-glucoside (orientin) and luteolin 6-C-glucoside (isoorientin) in the raw material. Their detection was conducted by comparing $R_{\mathrm{f}}$ coefficients using derivatization reagents and $366 \mathrm{~nm}$ UV light. In this work, the characteristic fluorescent bands after derivatization provided important clues for the identification of the major flavone present in the samples, while high performance liquid chromatography combined with ultraviolet spectrometry or a diode array detector (HPLC-UV/DAD) technique was employed for the simultaneous detection and quantification of these compounds [44]. 
Table 2. Thin layer chromatography in the analysis of luteolin derivatives.

\begin{tabular}{|c|c|c|c|c|c|}
\hline Luteolin Derivative & Stationary Phase & Mobile Phase & Detection & Analyzed Species & Ref. \\
\hline Luteolin & $\begin{array}{c}\text { silica gel } 60 \mathrm{~F}_{254} \\
\text { silica gel } 60 \mathrm{RP}-18 \mathrm{~F}_{254} \mathrm{~S}\end{array}$ & $\begin{array}{c}\text { Hx:EtOAc:AcOH }(31: 14: 5 v / v / v) ; \\
\text { To:DI:AcOH }(90: 25: 4 v / v / v) \\
\text { FA:H } \\
\text { OOMeOH }(5.5: 47.25: 47.25 v / v / v)\end{array}$ & $\begin{array}{c}\text { FBS; } \\
\text { UV, } 254,366 \mathrm{~nm}\end{array}$ & Artemisia annua & [25] \\
\hline $\begin{array}{l}\text { Luteolin 7-rutoside } \\
\text { Luteolin 7-rhamnoside }\end{array}$ & $\begin{array}{l}\text { silica gel } \\
\text { cellulose } \\
\text { polyamide }\end{array}$ & $\begin{array}{c}\text { EtOAc:FA:AcOH: } \mathrm{H}_{2} \mathrm{O}(100: 11: 11: 23 \\
v / v / v / v) \\
30 \% \mathrm{AcOH} \\
\text { CHCl } \mathrm{MeOH}: \mathrm{MEK}: \mathrm{AcAc}(9: 4: 2: 1 \mathrm{v} / v / v / v) ; \\
\text { To:MeOH:MEK:BuOH (300:150:150:3 } \\
v / v / v / v)\end{array}$ & $\begin{array}{l}\text { NP/PEG, aniline } \\
\text { phthalate; } \\
\text { UV, 254,366 nm } \\
\text { MS, NMR }\end{array}$ & Ligustrum vulgare & [26] \\
\hline $\begin{array}{l}\text { Luteolin 3'-glucoside } \\
\text { Luteolin 6,8-dihexoside } \\
\text { Luteolin 7-rutinoside }\end{array}$ & silica gel $60 \mathrm{~F}_{254}$ & $\begin{array}{c}\mathrm{MeOH}: \mathrm{H}_{2} \mathrm{O}(15: 5 \mathrm{v} / \mathrm{v}) ; \\
\mathrm{CHCl}_{3}: \mathrm{MeOH}(15: 5 \mathrm{v} / \mathrm{v}) ; \\
15 \% \mathrm{AcOH}\end{array}$ & $\begin{array}{l}\text { NP/PEG; } \\
\text { UV, } 366 \mathrm{~nm}\end{array}$ & $\begin{array}{c}\text { Phlomis persica } \\
\text { Ph. elliptica }\end{array}$ & [27] \\
\hline Luteolin 7-glucoside & $\begin{array}{c}\text { silica gel } \\
\text { polyamide plates }\end{array}$ & $\begin{array}{c}\text { EtOAc:FA: } \mathrm{H}_{2} \mathrm{O}(18: 1: 1 v / v / v) \\
\text { EtOAc:FA:AcOH:A } \mathrm{H}_{2} \mathrm{O}(100: 10: 10: 13 \\
v / v / v / v)\end{array}$ & $\begin{array}{l}\text { NP/PEG; } \\
\text { UV, 366 nm }\end{array}$ & Carduus acanthoides & [28] \\
\hline $\begin{array}{c}\text { Luteolin } \\
\text { Luteolin 7,4'-diglucoside } \\
\text { Luteolin } \\
\text { 6-hydroxy-7-glucoside } \\
\text { Luteolin 4'-glucoside } \\
\text { Luteolin 3'-glucoside } \\
\text { Luteolin 7-glucoside }\end{array}$ & silica gel $60 \mathrm{~F}_{254}$ & $\begin{array}{c}\text { EtOAc:Ace:FA }(8: 1: 1 \mathrm{v} / v / v) ; \\
\text { EtOAc: } \mathrm{H}_{2} \mathrm{O}: \text { AcOH:FA }(10: 3: 1: 1 \mathrm{v} / \mathrm{v} / \mathrm{v})\end{array}$ & $\begin{array}{l}\text { NP; } \\
\text { UV, IR, NMR }\end{array}$ & Leontopodium alpium & [29] \\
\hline $\begin{array}{c}\text { Luteolin } \\
\text { Luteolin 7-glucoside }\end{array}$ & silica gel $60 \mathrm{~F}_{254}$ & $\begin{array}{c}\text { To:Et }{ }_{2} \mathrm{O}: \mathrm{AcOH}(60: 40: 10 \mathrm{v} / \mathrm{v} / \mathrm{v}) \\
\text { EtOAc:AcOH:FA: } \mathrm{H}_{2} \mathrm{O}(100: 11: 11: 26\end{array}$ & $\begin{array}{l}\text { NP/PEG; } \\
\text { UV, } 366 \mathrm{~nm}\end{array}$ & $\begin{array}{l}\text { Matricaria recutita, Achillea millefolium, } \\
\text { Thymus vulgaris, Salvia officinalis }\end{array}$ & [30] \\
\hline
\end{tabular}

Abbreviations: AcAc, acetylacetone; Ace, acetone; $\mathrm{AcOH}$, acetic acid; $\mathrm{BuOH}$, butanol; $\mathrm{CHCl}_{3}$, chloroform; $\mathrm{DI}, 1$,4-dioxane; Et $\mathrm{O}_{2} \mathrm{O}$, diethyl ether; $\mathrm{EtOAc}$, ethyl acetate; $\mathrm{FA}$, formic acid; $\mathrm{FBS}$, Fast Blue B Salt; Hx, hexane; MeOH, methanol; MEK, methyl ethyl ketone; NP, $1 \%$ methanolic diphenylboric acid- $\beta$-ethylamino ester - natural product reagents; PEG, $5 \%$ ethanolic polyethylene glycol 4000; To, toluene; UV, ultraviolet spectroscopy; MS, mass spectrometry; NMR, nuclear magnetic resonance; IR, infrared. 
Table 3. High performance thin layer chromatography in the analysis of luteolin derivatives.

\begin{tabular}{|c|c|c|c|c|c|}
\hline Luteolin Derivative & Stationary Phase & Mobile Phase & Detection & Analyzed Species & Ref. \\
\hline \multirow{11}{*}{ Luteolin } & HPTLC silica gel $60 \mathrm{~F}_{254}$ & $\begin{array}{c}\text { Hx:EtOAc:AcOH } \\
\text { (31:14:5 v/v/v); } \\
\text { To:DI:AcOH } \\
(90: 25: 4 v / v / v)\end{array}$ & $\begin{array}{c}\text { FBS; } \\
\mathrm{UV}, 254,366 \mathrm{~nm}\end{array}$ & Artemisia anпиа & [25] \\
\hline & HPTLC diol $\mathrm{F}_{254} \mathrm{~S}$ & $\mathrm{CHCl}_{3}: \mathrm{Hx}: \mathrm{EtOAc}(34: 4 v / v / v)$ & $\begin{array}{r}2 \% \mathrm{AlCl}_{3} \\
\mathrm{UV}, 366 \mathrm{~nm}\end{array}$ & \multirow[t]{2}{*}{ Oxytropis glabra } & \multirow[t]{2}{*}[31]{} \\
\hline & $\begin{array}{l}\text { HPTLC silica gel } 60 \\
\text { RP-18W }\end{array}$ & $\begin{array}{c}\mathrm{BuOH}: \mathrm{MeOH}: \mathrm{H}_{2} \mathrm{O} \\
(300: 50: 50 \mathrm{v} / v /) \\
15 \% \mathrm{AcOH}\end{array}$ & $\begin{array}{l}\text { NPR/PEG; } \\
\text { UV, } 366 \mathrm{~nm}\end{array}$ & & \\
\hline & HPTLC silica gel $60 \mathrm{~F}_{254}$ & $\begin{array}{l}\text { EtOAc:MeOH:FA: } \mathrm{H}_{2} \mathrm{O} \\
\quad(20: 3: 1: 2 v / v / v / v)\end{array}$ & $\begin{array}{c}\mathrm{MeOH}: \mathrm{H}_{2} \mathrm{SO}_{4} \\
(95: 5 \mathrm{v} / \mathrm{v}) ; \\
\mathrm{UV}, 254,366 \mathrm{~nm}\end{array}$ & $\begin{array}{c}\text { Asparagus racemosus, } \\
\text { Withania somnifera, } \\
\text { Vitex negundo, } \\
\text { Plumbago zylenica, } \\
\text { Butea monosperma, } \\
\text { Tephrosia purpurea }\end{array}$ & [32] \\
\hline & HPTLC silica gel $60 \mathrm{~F}_{254}$ & $\begin{array}{l}\text { To:EtOAc:FA } \\
(10: 9: 1 v / v / v)\end{array}$ & $\mathrm{UV}, 254 \mathrm{~nm}$ & Cardiospermum halicacabum & {$[33]$} \\
\hline & HPTLC silica gel $60 \mathrm{~F}_{254}$ & $\begin{array}{l}\text { DCM:MeOH } \\
(70: 30 v / v)\end{array}$ & $\begin{array}{l}\text { UV, } 254 \mathrm{~nm} ; \\
\text { NMR }\end{array}$ & Satureja montana & [34] \\
\hline & HPTLC silica gel $\mathrm{G}_{60} \mathrm{~F}_{254}$ & $\begin{array}{l}\text { To:EtOAc:FA } \\
(6: 4: 1 \mathrm{v} / \mathrm{v} / \mathrm{v})\end{array}$ & $\mathrm{UV}, 349 \mathrm{~nm}$ & Hygrophila spinosa & {$[35]$} \\
\hline & HPTLC silica gel $60 \mathrm{~F}_{254}$ & $\begin{array}{l}\text { EtOAc:MeOH: } \mathrm{H}_{2} \mathrm{O}: \mathrm{AcOH} \\
(3: 1: 1: 1 v / v / v / v)\end{array}$ & $\mathrm{UV}, 254,366 \mathrm{~nm}$ & $\begin{array}{l}\text { Foeniculum vulgare, } \\
\text { Cuminum cyminum, } \\
\text { Apium graveolens, } \\
\text { Petroselinum crispum, } \\
\text { Anethum graveolens, } \\
\text { Ammi majus }\end{array}$ & [36] \\
\hline & HPTLC silica gel $60 \mathrm{~F}_{254}$ & $\begin{array}{l}\text { To:EtOAc:FA } \\
(3: 3: 0.8 v / v / v)\end{array}$ & $\begin{array}{c}\mathrm{MeOH}: \mathrm{H}_{2} \mathrm{SO}_{4} \\
(90: 10 \mathrm{v} / \mathrm{v}) \\
\mathrm{UV}, 254 \mathrm{~nm}\end{array}$ & Saraca asoca & [37] \\
\hline & HPTLC silica gel $60 \mathrm{~F}_{254}$ & $\begin{array}{l}\text { To:EtOAc:FA } \\
(6: 4: 0.3 v / v / v)\end{array}$ & $\begin{array}{l}\text { NP/PEG; } \\
\text { UV, } 366 \text { nm }\end{array}$ & Premna mucronata & {$[38]$} \\
\hline & HPTLC silica gel $G_{60} F_{254}$ & $\begin{array}{l}\text { To:EtOAc:FA } \\
(10: 9: 1 \mathrm{v} / \mathrm{v} / \mathrm{v})\end{array}$ & $\mathrm{UV}, 254,366 \mathrm{~nm}$ & Anisochilus carnosus & [39] \\
\hline
\end{tabular}


Table 3. Cont.

\begin{tabular}{|c|c|c|c|c|c|}
\hline Luteolin Derivative & Stationary Phase & Mobile Phase & Detection & Analyzed Species & Ref. \\
\hline \multirow{4}{*}{ Luteolin } & HPTLC silica gel 60 & $\begin{array}{l}n \text { Hx:EtOAc:FA } \\
(30: 20: 1.5 v / v / v)\end{array}$ & $\begin{array}{l}\text { NP/PEG; } \\
\mathrm{UV}, 349 \mathrm{~nm}\end{array}$ & Satureja hortensis & [40] \\
\hline & HPTLC silica gel $60 \mathrm{~F}_{254}$ & $\begin{array}{l}n \mathrm{Hx}: \mathrm{EtOAc:AcOH} \\
(5: 3: 1 v / v / v)\end{array}$ & UV & Propolis & [41] \\
\hline & HPTLC silica gel $60 \mathrm{GF}_{254}$ & $\begin{array}{c}\text { To:EtOAc:FA:MeOH } \\
(3: 3: 0.8: 0.2 v / v / v / v)\end{array}$ & $\mathrm{UV}, 254,365 \mathrm{~nm}$ & Eclipta alba & [42] \\
\hline & HPTLC silica gel $60 \mathrm{~F}_{254}$ & $\begin{array}{l}\text { To:EtOAc:FA } \\
(5: 3: 1 v / v / v)\end{array}$ & $\begin{array}{c}\mathrm{NP} ; \\
\mathrm{UV}, 366,254 \mathrm{~nm}\end{array}$ & Vitis vinifera & [43] \\
\hline \multirow{3}{*}{$\begin{array}{l}\text { Luteolin 6-glucoside } \\
\text { Luteolin 8-glucoside }\end{array}$} & HPTLC silica gel $60 \mathrm{~F}_{254}$ & $\begin{array}{c}\text { EtOAc:FA:AcOH: } \mathrm{H}_{2} \mathrm{O} \\
(100: 11: 11: 27 v / v / v)\end{array}$ & $\begin{array}{l}\text { NP/PEG; } \\
\text { UV, } 366 \mathrm{~nm}\end{array}$ & Clinacanthus nutans & [44] \\
\hline & HPTLC silica gel $60 \mathrm{~F}_{254}$ & $\begin{array}{c}\text { EtOAc:FA: } \mathrm{H}_{2} \mathrm{O} \\
(82: 9: 9 \mathrm{v} / \mathrm{v} / \mathrm{v})\end{array}$ & $\begin{array}{c}\text { NP/PEG; } \\
\text { UV, } 366 \mathrm{~nm}\end{array}$ & $\begin{array}{l}\text { Passiflora alata, } \\
\text { P. edulis }\end{array}$ & [45] \\
\hline & $\begin{array}{l}\text { Nano-DUASIL silica gel } \\
60\end{array}$ & $\begin{array}{l}\text { THF:To:FA: } \mathrm{H}_{2} \mathrm{O} \\
(16: 8: 2: 1 \mathrm{v} / v / v / v)\end{array}$ & $\mathrm{UV}, 350 \mathrm{~nm}$ & Phyllostachys pubescens & [46] \\
\hline \multirow{2}{*}{ Luteolin glucoside } & HPTLC silica gel $60 \mathrm{~F}_{254}$ & $\begin{array}{l}\text { EtOAc:FA:AcOH: } \mathrm{H}_{2} \mathrm{O} \\
(100: 11: 11: 26 \mathrm{v} / \mathrm{v} / \mathrm{v})\end{array}$ & $\begin{array}{l}\text { NP/PEG; } \\
\mathrm{UV}, 366 \mathrm{~nm}\end{array}$ & Equisetum arvense & [47] \\
\hline & HPTLC silica gel $60 \mathrm{~F}_{254}$ & $\begin{array}{l}\text { EtOAc:AcOH:FA: } \mathrm{H}_{2} \mathrm{O} \\
(10: 1.1: 1.1: 2.6 \mathrm{v} / \mathrm{v} / \mathrm{v})\end{array}$ & $\mathrm{UV}, 254,366 \mathrm{~nm}$ & Aerva javanica & [48] \\
\hline \multirow[t]{2}{*}{ Luteolin } & NP-HPTLC silica gel & $\begin{array}{c}\text { EtOAc:FA:AcOH: } \mathrm{H}_{2} \mathrm{O} \\
(100: 11: 11: 27 v / v / v / v) \\
\text { To:EtOAc:AcOH } \\
(50: 45: 5 v / v / v)\end{array}$ & $\begin{array}{c}\mathrm{H}_{2} \mathrm{O} \text { solution of } 4 \% \mathrm{Al}_{2}\left(\mathrm{SO}_{4}\right)_{3} \\
\mathrm{UV}, 365 \mathrm{~nm}\end{array}$ & Apis mellifera, honey & [49] \\
\hline & HPTLC silica gel $60 \mathrm{~F}_{254}$ & $\begin{array}{l}\text { To:EtFo:FA } \\
(6: 4: 1 v / v / v)\end{array}$ & & $\begin{array}{l}\text { Potentilla grandiflora, } \\
\text { P. recta, }\end{array}$ & \\
\hline Luteolin 7-glucoside & $\begin{array}{c}\text { modified HPTLC silica gel } \\
60 \mathrm{~F}_{254} \text { with } \mathrm{CN}, \mathrm{NH}_{2} \\
\text { HPTLC diol } \mathrm{F}_{254}\end{array}$ & $\begin{array}{c}\text { To:EtFo:FA } \\
\text { (7:5:1 v/v/v) } \\
\text { EtOAc:MEK:DIPE:FA } \\
(3: 10: 4: 1 v / v / v / v)\end{array}$ & $\begin{array}{c}\mathrm{NP} ; \\
\mathrm{UV}, 254,366 \mathrm{~nm}\end{array}$ & $\begin{array}{c}\text { P. anserina, } \\
\text { P. fruticose, } \\
\text { P. rupestris, } \\
\text { P. thuringiaca }\end{array}$ & [50] \\
\hline \multirow{2}{*}{ Luteolin 7-glucoside } & HPTLC silica gel 60 & $\begin{array}{l}\text { EtOAC:DCM:AcOH:FA: } \mathrm{H}_{2} \mathrm{O} \\
\quad(100: 25: 10: 10: 11 \mathrm{v} / \mathrm{v})\end{array}$ & $\begin{array}{c}\mathrm{NP} \\
\mathrm{UV}, 366 \mathrm{~nm}\end{array}$ & Lavandula stoechas & [51] \\
\hline & HPTLC silica gel $60 \mathrm{~F}_{254}$ & $\begin{array}{c}\text { EtOAc:AcOH:FA: } \mathrm{H}_{2} \mathrm{O} \\
(100: 11: 11: 26 v / v / v / v)\end{array}$ & $\begin{array}{c}\text { NP/PEG; } \\
\text { UV, 254, 366 nm }\end{array}$ & $\begin{array}{l}\text { Stachys sylvatica, } \\
\text { S. recta }\end{array}$ & [52] \\
\hline
\end{tabular}


Table 3. Cont.

\begin{tabular}{|c|c|c|c|c|c|}
\hline Luteolin Derivative & Stationary Phase & Mobile Phase & Detection & Analyzed Species & Ref. \\
\hline Luteolin 7-rutinoside & $\begin{array}{l}\text { HPTLC } \mathrm{NH}_{2} \\
\text { HPTLC RP-18W }\end{array}$ & $\begin{array}{c}\text { Ace: } \mathrm{AcOH} \\
(85: 15 v / v) \\
\mathrm{H}_{2} \mathrm{O}: \mathrm{MeOH} \\
(60: 40 v / v) \\
\end{array}$ & $\begin{array}{c}\text { MeOH: } \mathrm{AlCl}_{3}(98: 2 \mathrm{v} / \mathrm{v}), \\
\text { bis-diazodized sulfanilamide; } \\
\text { UV-VIS, } 365 \mathrm{~nm} \\
\text { IR, MS, NMR } \\
\end{array}$ & Mentha piperita & [53] \\
\hline $\begin{array}{l}\text { Luteolin 7-glucuronide } \\
\text { Luteolin 7-glucoside }\end{array}$ & $\begin{array}{c}\text { HPTLC silica gel } 60 \\
\text { HPTLC } \mathrm{NH}_{2}\end{array}$ & $\begin{array}{c}\text { Et:Ace:H2O:FA } \\
(55: 25: 10: 10 v / v / v / v) \\
\text { Ace:AcOH }(85: 15 v / v)\end{array}$ & $\begin{array}{c}\text { NP/PEG; } \\
\text { UV, } 365 \mathrm{~nm}\end{array}$ & $\begin{array}{l}\text { Mentha piperita, } \\
\text { Melissa officinalis, } \\
\text { Salvia officinalis }\end{array}$ & [54] \\
\hline $\begin{array}{l}\text { Luteolin 7-rutinoside } \\
\text { Luteolin 7-glucoside } \\
\text { Luteolin 7-glucuronide }\end{array}$ & $\begin{array}{c}\quad \text { HPTLC } \mathrm{NH}_{2} \\
\text { HPTLC silica gel } 60\end{array}$ & $\begin{array}{c}\text { Ace:AcOH }(85: 15 v / v) \\
\text { Ace:FA }(85: 15 v / v) \\
\text { DIPE:Ace: } \mathrm{H}_{2} \mathrm{O}: \mathrm{FA}(50: 30: 10: 10 \\
v / v / v / v)\end{array}$ & $\begin{array}{c}\mathrm{MeOH}: \mathrm{AlCl}_{3}(98: 2 \mathrm{v} / v), \mathrm{NP} / \mathrm{PEG} ; \\
\mathrm{UV}, 365 \mathrm{~nm}\end{array}$ & $\begin{array}{l}\text { Thymus vulgaris, } \\
\text { Th. serpyllum, } \\
\text { Majorana hortensis, } \\
\text { Mentha piperita }\end{array}$ & [55] \\
\hline $\begin{array}{c}\text { Luteolin } \\
\text { Luteolin acetyl hexuronide } \\
\text { Luteolin 7-rutinoside } \\
\text { Luteolin acetyl hexoside } \\
\text { Luteolin hexuronide }\end{array}$ & HPTLC silica gel $60 \mathrm{~F}_{254}$ & $\begin{array}{c}\text { pre-develop: } \mathrm{CHCl}_{3}: \mathrm{MeOH} \\
(1: 1 \mathrm{v} / v) ; \\
n \mathrm{Hx}: \mathrm{EtOAc:FA} \\
(20: 19: 1 \mathrm{v} / v / v)\end{array}$ & $\begin{array}{c}\text { NP: white light, } 254,366,330 \mathrm{~nm} ; \\
\text { PEG: } 254,297,340,366,430 \mathrm{~nm} ; \\
\text { paraffin- } n \text { Hx: } 254,320,360,366,400 \\
\text { nm; } \\
\text { ESI-MS/MS }\end{array}$ & $\begin{array}{c}\text { Rosmarinus officinalis } \\
\text { Salvia officinalis }\end{array}$ & [57] \\
\hline $\begin{array}{l}\text { Luteolin 3',7-diglucoside } \\
\text { Luteolin 4'-glucoside } \\
\text { Luteolin 7-glucoside } \\
\text { Luteolin 8-glucoside } \\
\text { Luteolin 6-glucoside } \\
\end{array}$ & HPTLC silica gel $60 \mathrm{~F}_{254}$ & $\begin{array}{l}\text { EtOAc:MeOH:AcOH:FA: } \mathrm{H}_{2} \mathrm{O} \\
(30: 1: 2: 1: 3 v / v / v / v / v)\end{array}$ & $\begin{array}{c}\mathrm{NP} ; \\
\mathrm{UV}, 366 \mathrm{~nm}\end{array}$ & Colocasia esculenta & [58] \\
\hline Luteolin 8-glucoside & $\begin{array}{c}\text { HPTLC RP } C_{18} \\
\text { HPTLC silica gel } F_{254}\end{array}$ & $\begin{array}{c}\text { FA:MeOH: } \mathrm{H}_{2} \mathrm{O} \\
(0.5: 6.65: 2.85 v / v / v) \\
\text { To:EtOAc:FA }(6: 5: 1 v / v / v)\end{array}$ & $\begin{array}{l}\text { ESI-MS/MS, } \\
254,366 \mathrm{~nm}\end{array}$ & Cyclanthera pedata & [59] \\
\hline
\end{tabular}

Abbreviations: Ace, acetone; $\mathrm{AcOH}$, acetic acid; $\mathrm{AlCl}_{3}$, aluminium trichloride; $\mathrm{Al}_{2}\left(\mathrm{SO}_{4}\right)_{3}$, aluminium sulfate; $\mathrm{BuOH}$, butanol; $\mathrm{CHCl}$, chloroform; $\mathrm{DCM}$, dichloromethane; $\mathrm{DI}$, 1,4-dioxane; DIPE, diisopropyl ether; ESI-MS/MS, electrospray ionization combined with tandem mass spectrometry; $\mathrm{EtFo}_{\mathrm{F}}$, ethyl formate; EtOAc, ethyl acetate; Et, ether; $\mathrm{FA}$, formic acid; $\mathrm{H}_{2} \mathrm{SO} \mathrm{O}_{4}$, sulfuric acid; $\mathrm{Hx}$, hexane; $\mathrm{MeOH}$, methanol; MEK, methyl ethyl ketone; $n \mathrm{Hx}, n$-hexane; NP, $1 \%$ methanolic diphenylboric acid- $\beta$-ethylamino ester - natural product reagents; neurotransmitters; PEG, 5\% ethanolic polyethylene glycol 4000; THF, tetrahydrofuran; To, toluene; UV-VIS, ultraviolet-visible spectroscopy; ESI-MS, electrospray ionization combined with mass spectrometry. 


\subsection{High Performance Liquid Chromatography in the Analysis of Luteolin Derivatives}

Among the many chromatographic methods, adsorptive chromatography, in which the mobile phase is liquid and the stationary phase is solid, is of the greatest practical importance. For example, this method has a much wider application than GC because it allows the analysis of substances in the form of liquids and soluble solids. Furthermore, it is also suitable for analysis of thermolabile substances because it is usually performed at low temperatures, and such samples are not degraded [61]. Due to the long analysis time, high mobile phase consumption and low efficiency, traditional CC is currently used mainly for preparative purposes. However, improved methods, such as HPLC and especially liquid chromatography combined with mass spectrometry (LC-MS), are increasingly used for analysis of natural compounds including luteolin derivatives [62].

High performance liquid chromatography has been performed since 1960. It is a dynamically developing method with a wide range of uses and has been proven to be very useful in phytochemical analysis. The principle of operation consists of pumping the mobile phase from the tank (or tanks) through the stationary phase-filled column. Eluents are previously filtered and degassed. Some systems are additionally equipped with thermostats that regulate the temperature of the column. If the chromatographic system is properly selected and applied, then the individual components are separated and detected. Strengthened signals are transmitted to the computer, where the obtained data are registered and properly processed [19]. The separation is based on competition of the molecules of the eluent and the substance being analyzed for the space on the adsorbent surface in the column $[63,64]$.

When choosing a column, one should be guided by the size of the sample, time of analysis and expected effect of the separation. Although columns with different diameters are available, those with a diameter of $4.6 \mathrm{~mm}$ are by far the most common. However, due to better detection of the components separated in smaller diameter columns, these columns are increasingly being used for separation and analysis $[19,63,65]$. In addition, improved separation can be achieved using a column filled with smaller particles. In addition to $5 \mu \mathrm{m}$ particles, which are most common, particles of $3 \mu \mathrm{m}$ in diameter or even smaller can be used. For example (Table 4), this approach was chosen when separating luteolin 2"-O-feruloylhexosyl-6-C-hexoside and luteolin 6-C-glucoside from Arenaria montana [66,67] and isoorientin in Achillea millefolium [68,69], as well as separating luteolin 6-C-hexosyl-8-C-pentoside, luteolin 2"-O-deoxyhexosyl-6-C-glucoside and luteolin 6-C-glucoside in Cymbopogon citratus [70,71]. Particles less than $2 \mu \mathrm{m}$ in diameter were used for ultra-performance liquid chromatography (UPLC) analysis of luteolin derivatives and other flavonoids in Lactuca sativa [72,73] and date palm (Phoenix dactylifera) [74].

Stationary phases with different polarity may be used in HPLC. In the normal phase system, polar column fillings are used, and most often the filling is silica gel. However, silica gel can adsorb water, which leads to the loss of the original separating properties of the column and thus to impaired reproducibility of the obtained results $[19,65]$. Therefore, the gel is often modified with the aim of enabling better separation of mixture components. This is performed mainly by bonding alkyl chains (or alkyl chains bearing other functional groups) to functional groups on the gel surface. Silica gels optimized in this way are referred to as the associated phase [2,65]. In the so-called reversed phase (RP) system, non-polar associated phases are used. Such systems are especially useful in the analysis of insoluble or poorly water-soluble compounds, as well as in the analysis of polar compounds, provided that a mobile phase with high water content is used. Typically, an octadecylsilane (ODS) phase, composed of 18 carbon atoms (RP-18), is employed. Such fillers can have different properties depending on the silica gel type and/or production method [19,65]. According to the available literature, the analysis of luteolin derivatives (Table 4) was performed exclusively with RP systems, and the RP-18 system was the most frequently used system, as exemplified in the separation of six luteolin derivatives in Securigera securidaca [75] or as many as ten derivatives in Capsicum annuum [76]. Stationary phases composed of 8 carbon atoms (RP-8) were rarely used. Examples include the separation of flavonoids in Coriandrum sativum [77] and Achillea millefolium [68,69]. 
The selection of an appropriate mobile phase is extremely important for chromatographic separation. The type of analyte, mixture composition, stationary phase and detector used should be taken into account. Mixtures of up to three components are most commonly used. In the reverse system, mixtures of $\mathrm{MeOH} / \mathrm{H}_{2} \mathrm{O}$ or acetonitrile $(\mathrm{ACN}) / \mathrm{H}_{2} \mathrm{O}$ are routinely used. As the amount of organic solvent increases, the retention time for non-polar substances decreases, while the addition of $\mathrm{H}_{2} \mathrm{O}$ extends their retention times. As a rule of thumb, systems containing $\mathrm{ACN} / \mathrm{H}_{2} \mathrm{O}$ eluents are more efficient than those containing $\mathrm{MeOH} / \mathrm{H}_{2} \mathrm{O}$ [19]. In the normal phase system, however, the base solvent is a non-polar eluent, and its polarity is appropriately modified by the addition of another solvent of higher polarity, e.g., chloroform $\left(\mathrm{CHCl}_{3}\right)$ [65]. According to the available literature, virtually all mobile phases used for analysis of luteolin derivatives consisted either of $\mathrm{ACN} / \mathrm{H}_{2} \mathrm{O}$, e.g., $[26,66,67,78]$ or, less often, $\mathrm{MeOH} / \mathrm{H}_{2} \mathrm{O}$ mixtures [72,79-81]. Additionally, a modifier such as FA, e.g., [79,82-84], $\mathrm{AcOH}$, e.g., [44,78,85-87], or trifluoroacetic acid (TFA) [88] was added to avoid peak tailing [89]. Analyses performed without acidic modifiers are rare [90].

In the chromatography process, the elution can be carried out in two ways. The first method is isocratic elution, which involves running with the same composition of mobile phase during the analysis. This means that a constant elution force is maintained throughout the entire separation period. In this case, even a slight change in the composition of the mobile phase may affect the results of the analysis. Isocratic elution is only rarely used for analysis of flavonoids. In the case of luteolin derivatives, this method was used only for analysis of luteolin 7-rhamnosyl- (1-6)-galactoside in Filago germanica [91], luteolin 7-rhamnosyl(1-6)galactoside in Galactites elegans [90], and various luteolin derivatives in Apium graveolens [80]. If the composition of the eluent changes during the division of the mixture, then the gradient elution can be described. The gradient can be linear or specifically programmed. With a change in the composition of the eluent, its elution force increases. For this reason, this method is used particularly for the separation of mixtures composed of substances of different polarity $[2,64]$. The vast majority of papers describing the separation of luteolin derivatives in plant mixtures use a gradient approach. Examples include the separation of luteolin 8-C-glucoside, luteolin 6-C-glucoside, luteolin 4'-O-glucoside and luteolin 7-O-glucoside from Chrysanthemum trifurcatum [92], thirteen luteolin derivatives from Cymbopogon citratus [70,71] and many others.

By far, the most common method for detection of luteolin derivatives is using diode array detectors (DADs) (also called photodiode array detectors, PDA), which were used in as many as 46 instances (e.g., for the analysis of luteolin derivatives and other flavonoids in Cymbopogon citratus [79], Dianthus versicolor [88], Clinacanthus nutans [44], Thymus alternans and others [93]). More often than not, however, DADs were combined with other detectors for additional structure determination or confirmation, with or without prior isolation. Examples of DADs combined with other detection methods for analysis of luteolin derivatives in plants include the use of tandem mass spectrometry (MS/MS) and NMR for Ligustrum vulgare [26], diode array detectors combined with electrospray ionization and mass spectrometry (DAD-ESI-MS) for Phoenix dactylifera [94] and Nerium indicum [95], electrospray ionization combined with tandem mass spectrometry (ESI-MS/MS) for Achillea moschata [96], electrospray ionization combined with time of flight mass spectrometry (ESI-TOF-MS) and electrospray ionization combined with ion trap and tandem mass spectrometry (ESI-IT-MS/MS) for Aloysia citrodora and many others.

For example, the HPLC-PAD-MS technique was used in the analysis of aerial flowering parts of the Edelweiss alpine region (Leontopodium alpinum). This method allowed the basic separation of almost all components of the L. alpinum extract prepared by exhaustive dichloromethane (DCM) followed by $\mathrm{MeOH}$ extraction. In total, 14 compounds have been isolated from the extract, including several luteolin derivatives. The authors used a gradient as the mobile phase as follows: $\mathrm{H}_{2} \mathrm{O}: 0.9 \%$ FA:0.1\% $\mathrm{AcOH}: 1.5 \% \mathrm{BuOH}(\mathrm{A})$ and $\mathrm{ACN}: 30 \% \mathrm{MeOH}: 0.9 \% \mathrm{FA}: 0.1 \% \mathrm{AcOH}(\mathrm{B})$ and $\mathrm{MeOH}(\mathrm{C})$. The structure of the isolated components was additionally confirmed using NMR spectroscopy [29]. 
Table 4. High performance liquid chromatography/ultra performance liquid chromatography in the analysis of luteolin derivatives

\begin{tabular}{|c|c|c|c|c|c|c|}
\hline Luteolin Derivative & $\begin{array}{c}\text { Stationary } \\
\text { Phase/Column }\end{array}$ & Mobile Phase & Conditions & Detection & Analyzed Species & Ref. \\
\hline \multirow{10}{*}{ Luteolin } & ODS & $0.2 \% \mathrm{PA}: \mathrm{H}_{2} \mathrm{O}(\mathrm{A}), \mathrm{MeOH}(\mathrm{B})$ & $\begin{array}{l}\text { injection volume: } 20 \mu \mathrm{L} \text {; } \\
\text { flow rate: } 1.0 \mathrm{~mL} / \mathrm{min}\end{array}$ & $\mathrm{DAD}, 360 \mathrm{~nm}$ & Honey & [97] \\
\hline & $\mathrm{ODSC}_{18}$ & $0.2 \% \mathrm{PA}: \mathrm{H}_{2} \mathrm{O}(58: 41 v / v)$ & $\begin{array}{l}\text { injection volume: } 50 \mu \mathrm{L} ; \\
\text { flow rate: } 1.0 \mathrm{~mL} / \mathrm{min}\end{array}$ & $\mathrm{UV}, 350 \mathrm{~nm}$ & $\begin{array}{l}\text { Chrysanthemum } \\
\text { morifolium }\end{array}$ & [98] \\
\hline & Kinetex $C_{18}$ & $\mathrm{ACN}(\mathrm{A}), 0.05 \%$ TFA: $\mathrm{H}_{2} \mathrm{O}(\mathrm{B})$ & flow rate: $0.8 \mathrm{~mL} / \mathrm{min}$ & $\mathrm{UV}, 254 \mathrm{~nm}$ & Enhalus acoroides & [99] \\
\hline & ODS Hypersil $C_{18}$ & $\begin{array}{c}\text { MeOH:ACN }(1.25: 1 v / v)(\mathrm{A}), 0.5 \% \\
\text { AcOH: } \mathrm{H}_{2} \mathrm{O} \text { (B) }\end{array}$ & flow rate: $0.8 \mathrm{~mL} / \mathrm{min}$ & MS & Perilla frutescens & {$[100]$} \\
\hline & Zorbax SB-C 18 & $\mathrm{PA}: \mathrm{H}_{2} \mathrm{O} \mathrm{pH} 4.0(\mathrm{~A}), \mathrm{ACN}(\mathrm{B})$ & $\begin{array}{l}\text { injection volume: } 50 \mu \mathrm{L} \text {; } \\
\text { flow rate: } 0.6 \mathrm{ml} / \mathrm{min} ; 25^{\circ} \mathrm{C}\end{array}$ & $\mathrm{DAD}, 330 \mathrm{~nm}$ & Vernonia condensata & [101] \\
\hline & $\mathrm{ODSC}_{18}$ & $\mathrm{MeOH}(\mathrm{A}), 0.05 \%$ TFA: $\mathrm{H}_{2} \mathrm{O}(\mathrm{B})$ & $\begin{array}{l}\text { injection volume: } 20 \mu \mathrm{L} \text {; } \\
\text { flow rate: } 1.0 \mathrm{~mL} / \mathrm{min}\end{array}$ & $\mathrm{UV}, 280 \mathrm{~nm}$ & Corchorus olitorius & [102] \\
\hline & $\mathrm{RP}$ & $0.5 \% \mathrm{PA}: \mathrm{H}_{2} \mathrm{O}(\mathrm{A}), \mathrm{ACN}(\mathrm{B})$ & $\begin{array}{c}\text { injection volume: } 20 \mu \mathrm{L} \text {; } \\
\text { flow rate: } 0.8 \mathrm{~mL} / \mathrm{min} ; \\
\text { at } 35^{\circ} \mathrm{C}\end{array}$ & Triple-TOF-MS & $\begin{array}{l}\text { Veronicastrum } \\
\text { latifolium }\end{array}$ & [103] \\
\hline & $\mathrm{RPC}_{18}$ & $\begin{array}{c}1 \% \text { FA: } \mathrm{H}_{2} \mathrm{O}(\mathrm{A}) \\
40 \% \text { solvent A:ACN (B) }\end{array}$ & $\begin{array}{l}\text { flow rate: } 0.5 \mathrm{~mL} / \mathrm{min} ; \\
25{ }^{\circ} \mathrm{C}\end{array}$ & DAD-ESI-MS/MS & Olea europaea, olive oil & [104] \\
\hline & Discovery $\mathrm{HS} \mathrm{C}_{18}$ & $2 \% \mathrm{AcOH}(\mathrm{A}), \mathrm{ACN}(\mathrm{B})$ & $\begin{array}{l}\text { injection volume: } 20 \mu \mathrm{L} \text {; } \\
\text { flow rate: } 0.8 \mathrm{~mL} / \mathrm{min}\end{array}$ & $\begin{array}{c}\text { DAD } \\
280,320 \mathrm{~nm}\end{array}$ & Agastache foeniculum & [105] \\
\hline & Ultrasphere $5 C_{18}$ & $\mathrm{H}_{2} \mathrm{O}(\mathrm{A}), \mathrm{ACN}(\mathrm{B})$ & $\begin{array}{l}\text { injection volume: } 10 \mu \mathrm{L} ; \\
\text { flow rate: } 1 \mathrm{~mL} / \mathrm{min} ; 25^{\circ} \mathrm{C}\end{array}$ & $\begin{array}{l}\text { ESI-MS; } \\
350 \mathrm{~nm}\end{array}$ & Rosa rugosa & [106] \\
\hline Luteolin 6-glucoside & Vydac RP $C_{18}$ & $\begin{array}{c}0.05 \% \text { TFA: } \mathrm{H}_{2} \mathrm{O}(\mathrm{A}), 0.038 \% \\
\text { TFA:ACN }(v / v)(\mathrm{B})\end{array}$ & $\begin{array}{l}\text { injection volume: } 10 \mu \mathrm{L} \\
\text { flow rate: } 1 \mathrm{~mL} / \mathrm{min} ; 36^{\circ} \mathrm{C}\end{array}$ & $\mathrm{UV}, 342 \mathrm{~nm}$ & Ficaria verna & [107] \\
\hline Luteolin 8-glucoside & $\mathrm{RPC}_{18}$ & $0.5 \% \mathrm{FA}: \mathrm{H}_{2} \mathrm{O}(\mathrm{A}), \mathrm{ACN}(\mathrm{B})$ & $\begin{array}{l}\text { injection volume: } 20 \mu \mathrm{L} \text {; } \\
\text { flow rate: } 0.5 \mathrm{~mL} / \mathrm{min} \text {; } \\
23{ }^{\circ} \mathrm{C}\end{array}$ & $\mathrm{DAD}, 254,340 \mathrm{~nm}$ & $\begin{array}{l}\text { Jatropha gossypiifolia, } J \text {. } \\
\text { mollissima }\end{array}$ & [108] \\
\hline \multirow[b]{2}{*}{ Luteolin 7-glucoside } & Hypersil gold $C_{18}$ & $0.1 \% \mathrm{FA}: \mathrm{H}_{2} \mathrm{O}(\mathrm{A}), 0.1 \% \mathrm{FA}: \mathrm{MeOH}(\mathrm{B})$ & $\begin{array}{c}\text { injection volume: } 10 \mu \mathrm{L} \text {; } \\
\text { flow rate: } 0.35 \mathrm{~mL} / \mathrm{min} \text {; } \\
38{ }^{\circ} \mathrm{C}\end{array}$ & MS & Rhoeo discolor & [109] \\
\hline & Thermo $C_{18}$ & $0.3 \% \mathrm{FA}: \mathrm{H}_{2} \mathrm{O}(\mathrm{A}), \mathrm{ACN}(\mathrm{B})$ & $\begin{array}{l}\text { injection volume: } 10 \mu \mathrm{L} \text {; } \\
\text { flow rate: } 1.0 \mathrm{~mL} / \mathrm{min} ; \\
30^{\circ} \mathrm{C}\end{array}$ & $\begin{array}{l}\text { DAD-Q- } \\
\text { Orbitrap-MS }\end{array}$ & $\begin{array}{c}\text { Epimedium } \\
\text { brevicornum, } \\
\text { Anacyclus pyrethrum, } \\
\text { Lycium barbarum, } \\
\text { Cuscuta australis }\end{array}$ & [110] \\
\hline
\end{tabular}


Table 4. Cont.

\begin{tabular}{|c|c|c|c|c|c|c|}
\hline Luteolin Derivative & $\begin{array}{c}\text { Stationary } \\
\text { Phase/Column }\end{array}$ & Mobile Phase & Conditions & Detection & Analyzed Species & Ref. \\
\hline Luteolin & Eclipse XDB $C_{18}$ & $\begin{array}{c}\text { 0.025\% AcOH: } \mathrm{H}_{2} \mathrm{O}(\mathrm{A}), 5 \% \\
\text { Ace: } \mathrm{ACN}(\mathrm{B})\end{array}$ & $30{ }^{\circ} \mathrm{C}$ & ESI-MS/MS & Olea europaea, olive oil & [111] \\
\hline Luteolin 7-glucoside & Eclipse XDB $C_{18}$ & $1 \% \mathrm{PA}: \mathrm{H}_{2} \mathrm{O}(\mathrm{A}), \mathrm{ACN}(\mathrm{B})$ & $\begin{array}{l}\text { injection volume: } 10 \mu \mathrm{L} \\
\text { flow rate: } 1 \mathrm{~mL} / \mathrm{min} ; 25^{\circ} \mathrm{C}\end{array}$ & DAD-ESI-QTOF-MS/MS & Verbascum ovalifolium & [112] \\
\hline $\begin{array}{l}\text { Luteolin 7-rutinoside } \\
\text { Luteolin 7-rhamnoside }\end{array}$ & Eclipse XDB $C_{18}$ & $\mathrm{ACN}(\mathrm{A}), 0.2 \% \mathrm{FA}(\mathrm{B})$ & $\begin{array}{l}\text { flow rate: } 0.3 \mathrm{~mL} / \mathrm{min} \text {; } \\
\quad 30^{\circ} \mathrm{C}\end{array}$ & $\begin{array}{l}\text { DAD, 230, 254, 290, } 334 \mathrm{~nm} ; \\
\text { MS/MS; NMR }\end{array}$ & Ligustrum vulgare & [26] \\
\hline $\begin{array}{c}\text { Luteolin } \\
\text { Luteolin 7,4-diglucoside } \\
\text { Luteolin 7-rutinoside } \\
\text { Luteolin 4-glucoside } \\
\text { Luteolin 3-glucoside } \\
\text { Luteolin 7-glucoside }\end{array}$ & Gemini $C_{18}$ & $0.5 \% \mathrm{AcOH}: \mathrm{H}_{2} \mathrm{O}(\mathrm{A}), \mathrm{ACN}(\mathrm{B})$ & $25^{\circ} \mathrm{C}$ & ESI-QTOF-MS & Olea europaea & [78] \\
\hline $\begin{array}{c}\text { Luteolin 6-glucoside } \\
\text { Luteolin 7-rhamnoside } \\
\text { Luteolin 7-glucoside } \\
\text { Luteolin 6-glucosyl-8-arabinoside }\end{array}$ & Spherisorb S5 ODS-2 & $5 \%$ FA: $\mathrm{H}_{2} \mathrm{O}(\mathrm{A}), \mathrm{MeOH}(\mathrm{B})$ & flow rate: $1 \mathrm{~mL} / \mathrm{min}$ & $\mathrm{DAD}, 280 \mathrm{~nm}$ & Cymbopogon citratus & [79] \\
\hline $\begin{array}{c}\text { Luteolin 2"-feruloylhexosyl-6-hexoside } \\
\text { Luteolin 6-glucoside }\end{array}$ & $\begin{array}{l}\text { Spherisorb S3 ODS-2 } \\
\mathrm{C}_{18}\end{array}$ & $0.1 \% \mathrm{FA}: \mathrm{H}_{2} \mathrm{O}(\mathrm{A}), \mathrm{ACN}(\mathrm{B})$ & $\begin{array}{l}\text { flow rate: } 0.5 \mathrm{~mL} / \mathrm{min} \text {; } \\
\qquad 35^{\circ} \mathrm{C}\end{array}$ & $\begin{array}{l}\text { DAD-MS; } \\
280,370 \mathrm{~nm}\end{array}$ & Arenaria montana & {$[66,67]$} \\
\hline $\begin{array}{c}\text { Luteolin } \\
\text { Luteolin 7-rutinoside } \\
\text { Luteolin 3-glucuronide } \\
\text { Luteolin 3'-(2"-acetyl)-glucuronide } \\
\text { Luteolin 6-hydroxy-7-glucoside }\end{array}$ & $\begin{array}{c}\text { Kinetex } 100 \mathrm{C}_{18} \\
\text { Superschera } 100 \mathrm{RP} \\
\mathrm{C}_{18}\end{array}$ & $1 \%$ FA: $\mathrm{H}_{2} \mathrm{O}(\mathrm{A}), 1 \%$ FA:ACN (B) & flow rate: $1 \mathrm{~mL} / \mathrm{min} ; 30^{\circ} \mathrm{C}$ & $\begin{array}{l}\text { ESI-QTOF-MS; } \\
\text { DAD, } 324 \mathrm{~nm}\end{array}$ & Rosmarinus officinalis & [82] \\
\hline $\begin{array}{l}\text { Luteolin hexosyl-hexoside-malylester } \\
\text { Luteolin 6-glucosyl-7-galactoside } \\
\text { Luteolin 6-glucosyl-7-rutinoside } \\
\text { Luteolin } \\
\text { 6-glucosyl-7-rhamnosyl-galactoside }\end{array}$ & AquasilW $\mathrm{C}_{18}$ & TFA solution (pH 2.8) (A), ACN (B) & $\begin{array}{l}\text { flow rate: } 1.0 \mathrm{~mL} / \mathrm{min} \text {; } \\
\qquad 5^{\circ} \mathrm{C}\end{array}$ & $\mathrm{UV} / \mathrm{DAD}, 340 \mathrm{~nm}$ & Dianthus versicolor & [88] \\
\hline $\begin{array}{l}\text { Luteolin 7-apiofuranosyl } \\
(1 \rightarrow 2) \text {-glucopyranoside } \\
\text { Luteolin 7-glucopyranoside } \\
\text { Luteolin 7-[apiofuranosyl } \\
(1 \rightarrow 2)-\left(6^{\prime \prime} \text {-malony)]-glucopyranoside }\right.\end{array}$ & Eclipse Plus $\mathrm{C}_{18}$ & $0.1 \%$ FA:MeOH (A), $0.1 \%$ FA: $\mathrm{H}_{2} \mathrm{O}(\mathrm{B})$ & $\begin{array}{c}\text { injection volume: } 10 \mu \mathrm{L} \text {; } \\
\text { isocratic mixture flow rate: } \\
0.3 \mathrm{~mL} / \mathrm{min}\end{array}$ & QTOF-MS/MS & Apium graveolens & [80] \\
\hline
\end{tabular}


Table 4. Cont

\begin{tabular}{|c|c|c|c|c|c|c|}
\hline Luteolin Derivative & $\begin{array}{c}\text { Stationary } \\
\text { Phase/Column }\end{array}$ & Mobile Phase & Conditions & Detection & Analyzed Species & Ref. \\
\hline $\begin{array}{l}\text { Luteolin 7-(6-rhamnosyl)hexoside } \\
\text { Luteolin 7-(2-rhamnosyl)hexoside } \\
\text { Luteolin } \\
\text { 7-(2-hexosyl[6-sulfate])hexoside } \\
\text { Luteolin 7-hexosyl(6-sulfate) }\end{array}$ & Kinetex $\mathrm{C}_{18}$ & $1 \% \mathrm{FA}(\mathrm{A}), \mathrm{ACN}(\mathrm{B})$ & $\begin{array}{l}\text { injection volume: } 8 \mu \mathrm{L} \text {; } \\
\text { flow rate: } 0.8 \mathrm{~mL} / \mathrm{min}\end{array}$ & DAD-ESI-MS; $340 \mathrm{~nm}$ & Phoenix dactylifera & [94] \\
\hline $\begin{array}{c}\text { Luteolin } \\
\text { Luteolin 7-glucoside }\end{array}$ & Gemini $C_{18}$ & $0.1 \%$ FA: $\mathrm{H}_{2} \mathrm{O}(\mathrm{A}), 0.1 \%$ FA:ACN (B) & $\begin{array}{l}0-60 \min , 10-60 \% \mathrm{~B} ; \\
\text { flow rate: } 1 \mathrm{~mL} / \mathrm{min}\end{array}$ & $\begin{array}{c}\text { DAD-ESI-MS/MS; } \\
210,270,310 \text { and } 350 \mathrm{~nm}\end{array}$ & Achillea moschata & [96] \\
\hline $\begin{array}{c}\text { Luteolin 7-glucoside } \\
\text { Luteolin 7,4'-diglucoside } \\
\text { Luteolin 6-hydroxy-7-glucoside } \\
\text { Luteolin 4'-glucoside } \\
\text { Luteolin 3'-glucoside }\end{array}$ & $\begin{array}{l}\text { Synergy Polar RP } 80 \AA \AA \text {, } \\
\text { LiChroCART 4-4 with } \\
\text { guard column } \\
\text { LiChrospher } 100 \mathrm{C}_{18}\end{array}$ & $\begin{array}{c}\text { 0.9\% FA:0.1\% AcOH:1.5\% } \\
\text { BuOH:H } \mathrm{H}_{2} \mathrm{O}(\mathrm{A}) \\
30 \% \text { MeOH:0.9\% FA:0.1\% } \\
\text { AcOH:ACN (B), } \\
\text { MeOH (C) }\end{array}$ & $\begin{array}{l}\text { injection volume: } 5 \mu \mathrm{L} \text {; } \\
\text { flow rate: } 1.0 \mathrm{~mL} / \mathrm{min} \text {; } \\
\quad 45^{\circ} \mathrm{C}\end{array}$ & $\begin{array}{l}\text { PAD; } \\
\text { MS; } \\
\text { UV; } \\
\text { IR; } \\
\text { NMR }\end{array}$ & Leontopodium alpium & [29] \\
\hline Luteolin 7-diglucuronide & Eclipse Plus $\mathrm{C}_{18}$ & $1 \% \mathrm{FA}: \mathrm{H}_{2} \mathrm{O}: \mathrm{ACN}(\mathrm{A}), \mathrm{ACN}(\mathrm{B})$ & $\begin{array}{l}\text { injection volume: } 20 \mu \mathrm{L} \text {; } \\
\text { flow rate: } 0.5 \mathrm{~mL} / \mathrm{min}\end{array}$ & $\begin{array}{l}\text { DAD; UV-VIS, 190-450 nm; } \\
\text { ESI-TOF-MS; } \\
\text { ESI-IT-MS/MS }\end{array}$ & Lippia citrodora & [113] \\
\hline $\begin{array}{l}\text { Luteolin 5-rutinoside } \\
\text { Luteolin 7-rutinoside }\end{array}$ & Luna $C_{18}$ & $0.1 \%$ FA: $\mathrm{H}_{2} \mathrm{O}(\mathrm{A}), \mathrm{ACN}(\mathrm{B})$ & $\begin{array}{l}\text { injection volume: } 5 \mu \mathrm{L} \text {; } \\
\text { flow rate: } 1.0 \mathrm{~mL} / \mathrm{min}\end{array}$ & $\begin{array}{l}\text { DAD, } 200-400 \mathrm{~nm} ; \\
\text { ESI-MS }\end{array}$ & Nerium indicum & [95] \\
\hline $\begin{array}{l}\text { Luteolin diglucoside } \\
\text { Luteolin 7-rutinoside } \\
\text { Luteolin 7-glucoside }\end{array}$ & Luna $\mathrm{C}_{18}$ & $1 \% \mathrm{FA}: \mathrm{H}_{2} \mathrm{O}(\mathrm{A}), \mathrm{ACN}(\mathrm{B})$ & $\begin{array}{l}\text { injection volume: } 20 \mu \mathrm{L} \text {; } \\
\quad \text { flow rate: } 1 \mathrm{~mL} / \mathrm{min}\end{array}$ & PDA-ESI-MS/MS & Taraxacum officinale & [114] \\
\hline $\begin{array}{c}\text { Luteolin } \\
\text { Luteolin 2"-Galloyl-4'-glucoside } \\
\text { Luteolin 4'-glucoside }\end{array}$ & Gemini RP $\mathrm{C}_{18}$ & $2 \% \mathrm{AcOH}: \mathrm{H}_{2} \mathrm{O}(\mathrm{A}), \mathrm{ACN}(\mathrm{B})$ & - & $\begin{array}{c}\text { ESI-MS; } \\
257,278 \text { and } 340 \mathrm{~nm}\end{array}$ & Pistacia atlantica & [85] \\
\hline $\begin{array}{c}\text { Luteolin } \\
\text { 6-methoxy-8-arabinosyl-7-glucoside } \\
\text { Luteolin 8-glucoside } \\
\text { Luteolin 8-rhamnosyl-7-rhamnoside }\end{array}$ & $\begin{array}{c}\text { Symmetry Shield } \\
\text { Waters } \mathrm{RP}_{18} \\
\text { Symmetry Shield } \\
\mathrm{RP}_{18}\end{array}$ & $0.2 \% \mathrm{FA}: \mathrm{H}_{2} \mathrm{O}(\mathrm{A}), \mathrm{ACN}(\mathrm{B})$ & flow rate: $1.2 \mathrm{~mL} / \mathrm{min}$ & $\begin{array}{l}\text { UV/PAD-MS; UV; } \\
\text { 200-600 nm } \\
\text { UV/PAD }\end{array}$ & Saccharum officinarum & [115] \\
\hline Luteolin 8-glucoside & Kinetex PFP & $\begin{array}{c}0.8 \% \text { AcOH: } \mathrm{H}_{2} \mathrm{O}(\mathrm{A}) \\
\mathrm{ACN}(\mathrm{B})\end{array}$ & $\begin{array}{l}\text { injection volume: } 10 \mu \mathrm{L} \text {; } \\
\text { flow rate: } 0.7 \mathrm{~mL} / \mathrm{min} \text {; } \\
\qquad 40^{\circ} \mathrm{C}\end{array}$ & UV/DAD, $330 \mathrm{~nm}$ & Clinacanthus nutans & [44] \\
\hline
\end{tabular}


Table 4. Cont

\begin{tabular}{|c|c|c|c|c|c|c|}
\hline Luteolin Derivative & $\begin{array}{c}\text { Stationary } \\
\text { Phase/Column }\end{array}$ & Mobile Phase & Conditions & Detection & Analyzed Species & Ref. \\
\hline $\begin{array}{c}\text { Luteolin } \\
\text { Luteolin 7-glucoside } \\
\text { Luteolin 7-glucuronyl-3-glucoside } \\
\text { Luteolin 6-glucoside } \\
\text { Luteolin 6-glucosyl-2"-rhamnoside } \\
\text { Luteolin 6-glucosyl-4'-glucoside } \\
\text { Luteolin 3'-glucoside }\end{array}$ & Eclipse XDB $\mathrm{C}_{18}$ & $\mathrm{MeOH}(\mathrm{A}), 0.2 \%$ FA: $\mathrm{H}_{2} \mathrm{O}(\mathrm{B})$ & $\begin{array}{l}\text { flow rate: } 0.8 \mathrm{~mL} / \mathrm{min} \text {; } \\
\qquad 30^{\circ} \mathrm{C}\end{array}$ & $\begin{array}{l}\text { DAD-MS/MS; } \\
\text { UV; } \\
254,360 \mathrm{~nm}\end{array}$ & Securigera securidaca & [75] \\
\hline $\begin{array}{l}\text { Luteolin hexosyl-rhamnoside } \\
\text { Luteolin 7-rutinoside } \\
\text { Luteolin 7-glucoside }\end{array}$ & $\begin{array}{c}\text { Spherisorb S3 ODS-2 } \\
\mathrm{C}_{8}\end{array}$ & $0.1 \% \mathrm{FA}: \mathrm{H}_{2} \mathrm{O}(\mathrm{A}), \mathrm{ACN}(\mathrm{B})$ & $\begin{array}{l}\text { flow rate: } 0.5 \mathrm{~mL} / \mathrm{min} \text {; } \\
\qquad 35^{\circ} \mathrm{C}\end{array}$ & $\begin{array}{l}\text { DAD-MS; } \\
\text { 280,370 nm }\end{array}$ & Coriandrum sativum & [77] \\
\hline $\begin{array}{c}\text { Luteolin } \\
\text { Luteolin 6-glucosyl-8-arabinoside } \\
\text { Luteolin 7-glucoside } \\
\text { Luteolin 6-arabinosyl-8-glucoside }\end{array}$ & XDB $C_{18}$ & $1 \% \mathrm{FA}: \mathrm{H}_{2} \mathrm{O}(\mathrm{A}), \mathrm{ACN}(\mathrm{B})$ & $\begin{array}{l}\text { injection volume: } 100 \mu \mathrm{L} \text {; } \\
\text { flow rate: } 4 \mathrm{~mL} / \mathrm{min}\end{array}$ & $\begin{array}{l}\text { NMR; } \\
\text { MS }\end{array}$ & Casimiroa edulis & {$[116,117]$} \\
\hline $\begin{array}{c}\text { Luteolin 3-glucopyranoside } \\
\text { Luteolin 7-glucopyranoside } \\
\text { Luteolin 7-rutinoside } \\
\text { Luteolin methoxy-hexoside } \\
\text { Luteolin 4'-glucopyranoside }\end{array}$ & PLRP-S $100 \AA ̊$ & $1 \% \mathrm{FA}: \mathrm{H}_{2} \mathrm{O}(\mathrm{A}), \mathrm{ACN}(\mathrm{B})$, & $\begin{array}{l}\text { injection volume: } 100 \mu \mathrm{L} \text {; } \\
\text { flow rate: } 1.5 \mathrm{~mL} / \mathrm{min} \\
\text { injection volume: } 100 \mu \mathrm{L} \text {; } \\
\text { flow rate: } 4 \mathrm{~mL} / \mathrm{min}\end{array}$ & MS & Thymus alternans & [93] \\
\hline Luteolin 6-hydroxy-7-glucoside & Gemini $C_{18}$ & $\begin{array}{c}0.1 \% \text { FA: } \mathrm{H}_{2} \mathrm{O}(\mathrm{A}) \\
\text { ACN (B) }\end{array}$ & flow rate: $0.8 \mathrm{~mL} / \mathrm{min}$ & $\begin{array}{c}\text { UV-VIS, 200-400 nm; } \\
\text { MS; } \\
\text { DAD; } \\
\text { NMR }\end{array}$ & Athrixia phylicoides & [118] \\
\hline $\begin{array}{c}\text { Luteolin } \\
\text { Luteolin 7-rhamnoside }\end{array}$ & Syncronis $\mathrm{C}_{18}$ & $0.1 \% \mathrm{AcOH}: \mathrm{H}_{2} \mathrm{O}(\mathrm{A}), \mathrm{ACN}(\mathrm{B})$ & $\begin{array}{l}\text { injection volume: } 5 \mu \mathrm{L} \text {; } \\
\text { flow rate: } 0.3 \mathrm{~mL} / \mathrm{min}\end{array}$ & DAD-MS/MS & Honey & [86] \\
\hline $\begin{array}{l}\text { Luteolin } \\
\text { Luteolin rhamnosyl hexoside } \\
\text { Luteolin rhamnosyl dihexoside }\end{array}$ & HSS T3 & $0.1 \%$ FA: $\mathrm{H}_{2} \mathrm{O}(\mathrm{A}), 0.1 \%$ FA:ACN (B) & $\begin{array}{l}\text { injection volume: } 3.1 \mu \mathrm{L} \text {; } \\
\text { flow rate: } 0.15 \mathrm{~mL} / \mathrm{min}\end{array}$ & PDA-MS & Phoenix dactylifera & [74] \\
\hline $\begin{array}{l}\text { Luteolin diglucuronide } \\
\text { Luteolin 7-glucuronide } \\
\text { Luteolin 7-rutinoside }\end{array}$ & S3 ODS- $2 \mathrm{C}_{18}$ & $0.1 \% \mathrm{FA}: \mathrm{H}_{2} \mathrm{O}(\mathrm{A}), \mathrm{ACN}(\mathrm{B})$ & $\begin{array}{l}\text { flow rate: } 0.5 \mathrm{~mL} / \mathrm{min} \text {; } \\
\qquad 35^{\circ} \mathrm{C} .\end{array}$ & $\begin{array}{l}\text { DAD-ESI-MS; } \\
370,330 \text { and } \\
280 \mathrm{~nm}\end{array}$ & $\begin{array}{l}\text { Thymus pallescens, } \\
\text { Saccocalyx satureioides, }\end{array}$ & {$[119,120]$} \\
\hline $\begin{array}{l}\text { Luteolin 7-glucoside } \\
\text { Luteolin 6-glucoside }\end{array}$ & & & & & $\begin{array}{l}\text { Ptychotis verticillata } \\
\text { Coleostephus myconis }\end{array}$ & \\
\hline
\end{tabular}


Table 4. Cont.

\begin{tabular}{|c|c|c|c|c|c|c|}
\hline Luteolin Derivative & $\begin{array}{c}\text { Stationary } \\
\text { Phase/Column }\end{array}$ & Mobile Phase & Conditions & Detection & Analyzed Species & Ref. \\
\hline $\begin{array}{c}\text { Luteolin } \\
\text { Luteolin rutinoside } \\
\text { Luteolin hexoside } \\
\text { Luteolin 6-hexoside } \\
\end{array}$ & Kinetex $C_{18}$ & $\mathrm{ACN}(\mathrm{A}), 0.1 \% \mathrm{FA}: \mathrm{H}_{2} \mathrm{O}(\mathrm{B})$ & $\begin{array}{l}\text { injection volume: } 10 \mu \mathrm{L} \text {; } \\
\text { flow rate: } 0.4 \mathrm{~mL} / \mathrm{min}\end{array}$ & ESI-MS & $\begin{array}{c}\text { Lathyrus pratensis } \\
\text { L.aureus }\end{array}$ & {$[121]$} \\
\hline $\begin{array}{l}\text { Luteolin 7-glucoside } \\
\text { Luteolin hexuronide }\end{array}$ & Luna $\mathrm{C}_{18}$ & $\begin{array}{l}5 \% \mathrm{FA}: \mathrm{H}_{2} \mathrm{O}(\mathrm{A}), \mathrm{MeOH}(\mathrm{B}) \\
0.1 \% \mathrm{FA}: \mathrm{H}_{2} \mathrm{O}(\mathrm{A}), \mathrm{MeOH}(\mathrm{B})\end{array}$ & $\begin{array}{l}\text { injection volume: } 100 \mu \mathrm{L} ; \\
\text { flow rate: } 1 \mathrm{~mL} / \mathrm{min} ; 35^{\circ} \mathrm{C} \\
\text { injection volume: } 10 \mu \mathrm{L} ; \\
\text { flow rate: } 0.5 \mathrm{~mL} / \mathrm{min} ; \\
40^{\circ} \mathrm{C}\end{array}$ & $\begin{array}{c}\text { DAD, } 200,600 \mathrm{~nm} \\
\text { ESI-MS/MS }\end{array}$ & Thymus pulegioides & [81] \\
\hline $\begin{array}{l}\text { Luteolin } \\
\text { Luteolin hexosyl-pentoside } \\
\text { Luteolin 6-glucoside } \\
\text { Luteolin 8-glucoside } \\
\text { Luteolin 7-glucoside }\end{array}$ & Eclipse Plus $\mathrm{C}_{18}$ & $0.5 \%$ AcOH: $\mathrm{H}_{2} \mathrm{O}(\mathrm{A}), \mathrm{ACN}(\mathrm{B})$ & $\begin{array}{l}\text { injection volume: } 5 \mu \mathrm{L} \text {; } \\
\text { flow rate: } 0.5 \mathrm{~mL} / \mathrm{min}\end{array}$ & $\begin{array}{l}\text { DAD-QTOF-MS; } \\
\text { 325-371 nm }\end{array}$ & Ficus carica & [87] \\
\hline $\begin{array}{c}\text { Luteolin } \\
\text { (3-hydroxy-3-methylglutaroyl)-X"-deox } \\
\text { Luteolin 6-hexoside } \\
\text { Luteolin 6-rutinoside } \\
\text { Luteolin dihexoside }\end{array}$ & $\begin{array}{l}\text { osplaf } \times 2 \text { Gide with } \\
\text { ODS- } 2 \mathrm{C}_{18} \text { guard } \\
\text { cartridge }\end{array}$ & $1 \% \mathrm{FA}: \mathrm{H}_{2} \mathrm{O}(\mathrm{A}), \mathrm{MeOH}(\mathrm{B})$ & $\begin{array}{l}\text { flow rate: } 0.2 \mathrm{~mL} / \mathrm{min} \text {; } \\
\qquad 25^{\circ} \mathrm{C}\end{array}$ & PDA-ESI-MS & Urtica membranacea & {$[122]$} \\
\hline $\begin{array}{c}\text { Luteolin } \\
\text { Luteolin 6,8-dihexoside } \\
\text { Luteolin 6-hexosyl-8-pentoside } \\
\text { Luteolin 6-pentosyl-8-hexoside } \\
\text { Luteolin 6-hexoside } \\
\text { Luteolin 8-hexoside } \\
\text { Luteolin } \\
\text { 7-(2"-pentosyl-4"-hexosyl)hexoside } \\
\text { Luteolin 7-(2"-pentosyl)hexoside } \\
\text { Luteolin 7-glucoside } \\
\text { Luteolin } \\
\text { 7-[2"-(5"'--sinapoyl)pentosyl]hexoside } \\
\text { Luteolin 7-(2"-pentosyl-4"-hexosyl- } \\
\text { 6"-malonyl)hexoside }\end{array}$ & Syncronis $\mathrm{C}_{18}$ & $0.01 \% \mathrm{AcOH}: \mathrm{H}_{2} \mathrm{O}(\mathrm{A}), \mathrm{ACN}(\mathrm{B})$ & $\begin{array}{l}\text { inaction volume: } 5 \mu \mathrm{L} ; \\
\text { flow rate: } 0.25 \mathrm{~mL} / \mathrm{min}\end{array}$ & MS & Capsicum aпnиит & [76] \\
\hline $\begin{array}{l}\text { Luteolin 7-(2"-pentosyl-6"'-malonyl) } \\
\text { hexoside } \\
\text { Luteolin 7-[2"-(5"'-sinapoyl)pentosyl- } \\
\text { 6"-malonyl]hexoside }\end{array}$ & & & & & & \\
\hline
\end{tabular}


Table 4. Cont.

\begin{tabular}{|c|c|c|c|c|c|c|}
\hline Luteolin Derivative & $\begin{array}{c}\text { Stationary } \\
\text { Phase/Column }\end{array}$ & Mobile Phase & Conditions & Detection & Analyzed Species & Ref. \\
\hline $\begin{array}{c}\text { Luteolin } \\
\text { Luteolin 6,8-diglucoside } \\
\text { Luteolin 8-glucoside } \\
\text { Luteolin 7-rutinoside }\end{array}$ & Purospher star $\mathrm{C}_{18}$ & $\begin{array}{l}10 \% \text { FA: } \mathrm{H}_{2} \mathrm{O}(\mathrm{A}) \\
\text { ACN (B) }\end{array}$ & $25^{\circ} \mathrm{C}$ & $\begin{array}{l}\text { DAD-ESI-MS; } \\
320-280 \mathrm{~nm}\end{array}$ & Citrus aurantifolia & [123] \\
\hline $\begin{array}{c}\text { Luteolin } \\
\text { Luteolin 7-glucoside } \\
\text { Luteolin 7-rutinoside }\end{array}$ & Zorbax SB-C 18 & $\begin{array}{l}\mathrm{AFNH}_{4}: \mathrm{H}_{2} \mathrm{O}: \mathrm{ACN}: \mathrm{FA}(\mathrm{A}), \\
\mathrm{AFNH}_{4}: \mathrm{H}_{2} \mathrm{O}: \mathrm{ACN}: \mathrm{FA} \mathrm{(B)}\end{array}$ & $\begin{array}{l}\text { injection volume: } 5 \mu \mathrm{L} ; \\
\text { flow rate: } 1.0 \mathrm{~mL} / \mathrm{min}\end{array}$ & $\begin{array}{l}\text { ESI-MS; } \\
325 \mathrm{~nm}\end{array}$ & Caucalis platycarpos & [124] \\
\hline $\begin{array}{l}\text { Luteolin 7-glucoside } \\
\text { Luteolin 7-glucuronide } \\
\text { Luteolin 7-rutinoside }\end{array}$ & Beta-Basic $\mathrm{C}_{18}$ & $5 \%$ FA:ACN (A); $5 \%$ FA: $\mathrm{H}_{2} \mathrm{O}(\mathrm{B})$ & $\begin{array}{l}\text { injection volume: } 20 \mu \mathrm{L} \text {; } \\
\text { flow rate: } 0.9 \mathrm{~mL} / \mathrm{min}\end{array}$ & $\mathrm{UV}, 280 \mathrm{~nm}$ & $\begin{array}{l}\text { Melissa officinalis } \\
\text { Mentha piperita, } \\
\text { Salvia officinalis }\end{array}$ & [54] \\
\hline \multirow{2}{*}{$\begin{array}{c}\text { Luteolin } \\
\text { Luteolin 7-glucuronide } \\
\text { Luteolin 7-rhamnosyl-hexoside } \\
\text { Luteolin 7-rutinoside } \\
\text { Luteolin 7-glucoside }\end{array}$} & $\begin{array}{c}\text { UPLC BEH } \mathrm{C}_{18} \text { and a } \\
\text { Acquity UPLC BEH } \\
\mathrm{C}_{18} \text { VanGuardTM } \\
\text { pre-column }\end{array}$ & $\begin{array}{l}\text { 0.1\% AcOH: } \mathrm{H}_{2} \mathrm{O}(\mathrm{A}) \text {, } \\
0.1 \% \mathrm{AcOH}: \mathrm{MeOH}(\mathrm{B})\end{array}$ & $\begin{array}{l}\text { injection volume: } 5 \mu \mathrm{L} \text {; } \\
\text { flow rate: } 0.5 \mathrm{~mL} / \mathrm{min}\end{array}$ & $\begin{array}{l}\text { DAD-ESI-QTOF-MS; } \\
370 \mathrm{~nm}\end{array}$ & \multirow[t]{2}{*}{ Lactuca sativa } & [72] \\
\hline & HSS T3 & $0.1 \%$ FA: $\mathrm{H}_{2} \mathrm{O}(\mathrm{A}), 0.1 \% \mathrm{FA}: \mathrm{ACN}(\mathrm{B})$ & $\begin{array}{l}\text { injection volume: } 3 \mu \mathrm{L} \text {; } \\
\text { flow rate: } 0.4 \mathrm{~mL} / \mathrm{min}\end{array}$ & IMS-QTOF-MS & & [73] \\
\hline 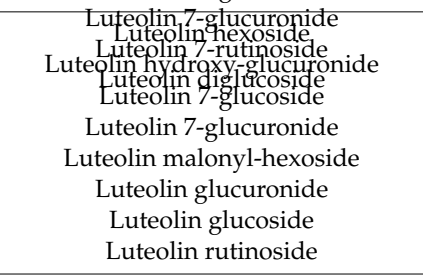 & Hypersil Gold $\mathrm{C}_{18}$ & $0.1 \% \mathrm{FA}: \mathrm{H}_{2} \mathrm{O}(\mathrm{A}), \mathrm{ACN}(\mathrm{B})$ & flow rate: $0.2 \mathrm{~mL} / \mathrm{min}$ & DAD-ESI-MS & $\begin{array}{c}\text { Salvia elegans, } \\
\text { S. greggii, } \\
\text { S. officinalis } \\
\\
\text { Thymus barona, } \\
\text { T. pseudolanuginosus, }\end{array}$ & {$[125,126]$} \\
\hline Luteolin 7-rhamnosyl(1-6)galactoside & Eclipse XDB- $C_{18}$ & $0.1 \%$ FA: $\mathrm{H}_{2} \mathrm{O}(\mathrm{A}), 0.1 \% \mathrm{FA}: \mathrm{ACN}(\mathrm{B})$ & $\begin{array}{l}\text { flow rate: } 0.5 \mathrm{~mL} / \mathrm{min} ; \\
25^{\circ} \mathrm{C}\end{array}$ & MS & $\begin{array}{l}\text { T. caespitititus } \\
\text { Filago germanica }\end{array}$ & [91] \\
\hline Luteolin 4'-glucuronide & $\mathrm{C}_{18} \mu$-Bondapak $\mathrm{RP}_{18}$ & $\mathrm{MeOH}: \mathrm{H}_{2} \mathrm{O}(42: 58 v / v)$ & flow rate: $2.0 \mathrm{~mL} / \mathrm{min}$ & NMR & Galactites elegans & [90] \\
\hline $\begin{array}{c}\text { Luteolin } \\
\text { Luteolin 3'-7-diglucoside } \\
\text { Luteolin 7-glucoside } \\
\text { Luteolin 6-(2-rhamnosyl)-hexoside } \\
\text { Luteolin (pentosyl)-hexoside } \\
\text { Luteolin hexoside }\end{array}$ & Kinetex $100 \mathrm{~A} \mathrm{C}_{18}$ & $1 \% \mathrm{FA}: \mathrm{H}_{2} \mathrm{O}(\mathrm{A}), \mathrm{ACN}(\mathrm{B})$ & $\begin{array}{l}\text { injection volume: } 5 \mu \mathrm{L} \text {; } \\
\text { flow rate: } 0.8 \mathrm{~mL} / \mathrm{min} \text {; } \\
\qquad 25^{\circ} \mathrm{C}\end{array}$ & $\begin{array}{l}\text { DAD, } 340 \mathrm{~nm} \\
\text { DAD-ESI-MS/MS; } \\
\text { 280, } 340 \mathrm{~nm}\end{array}$ & Allophylus africanus & {$[83,127]$} \\
\hline Luteolin 6-glucoside & ODS2 $C_{8}$ & $0.1 \% \mathrm{FA}: \mathrm{H}_{2} \mathrm{O}(\mathrm{A}), \mathrm{ACN}(\mathrm{B})$ & $\begin{array}{l}\text { flow rate: } 0.5 \mathrm{~mL} / \mathrm{min} \text {; } \\
\qquad 35^{\circ} \mathrm{C}\end{array}$ & $\begin{array}{l}\text { DAD-ESI-MS; } \\
280,370 \mathrm{~nm}\end{array}$ & Achillea millefolium & {$[68,69]$} \\
\hline
\end{tabular}


Table 4. Cont.

\begin{tabular}{|c|c|c|c|c|c|c|}
\hline Luteolin Derivative & $\begin{array}{c}\text { Stationary } \\
\text { Phase/Column }\end{array}$ & Mobile Phase & Conditions & Detection & Analyzed Species & Ref. \\
\hline $\begin{array}{c}\text { Luteolin 6-hexosyl-8-pentoside } \\
\text { Luteolin 2"-deoxyhexosyl-6-glucoside } \\
\text { Luteolin 6-glucoside } \\
\text { Luteolin 6-pentosyl-8-pentoside } \\
\text { Luteolin-7-rhamnoside } \\
\text { Luteolin 7-glucoside } \\
\text { Luteolin 2"-deoxyhexosyl-pentoside } \\
\text { Luteolin 6-pentoside } \\
\text { Luteolin 2"-deoxyosyl-6-(6-deoxy- } \\
\text { pento-hexosuloside }\end{array}$ & S3 ODS-2 $\mathrm{C}_{18}$ & $0.1 \%$ FA: $\mathrm{H}_{2} \mathrm{O}(\mathrm{A}), \mathrm{ACN}(\mathrm{B})$ & $\begin{array}{l}\text { flow rate: } 0.5 \mathrm{~mL} / \mathrm{min} \text {; } \\
\qquad 35^{\circ} \mathrm{C}\end{array}$ & $\begin{array}{l}\text { DAD-MS; } \\
280,370 \mathrm{~nm}\end{array}$ & Cymbopogon citratus & {$[70,71]$} \\
\hline $\begin{array}{l}\text { Luteolin } \\
\text { Luteolin 8-glucoside } \\
\text { Luteolin 4'-glucoside } \\
\text { Luteolin 7-glucoside } \\
\text { Luteolin 6-glucoside }\end{array}$ & $\mathrm{C}_{18}$ & $0.005 \% \mathrm{FA}: \mathrm{H}_{2} \mathrm{O}(\mathrm{A}), \mathrm{MeOH}(\mathrm{B})$ & $\begin{array}{l}\text { injection volume: } 10 \mu \mathrm{L} \text {; } \\
\text { flow rate: } 0.5 \mathrm{~mL} / \mathrm{min} \text {; } \\
\qquad 30^{\circ} \mathrm{C}\end{array}$ & MS-Orbitrap & $\begin{array}{l}\text { Chrysanthemum } \\
\text { trifurcatum }\end{array}$ & [92] \\
\hline $\begin{array}{c}\text { Luteolin 6-glucoside } \\
\text { Luteolin 8-glucoside } \\
\text { Luteolin 6-deoxyhexosyl-8-pentoside } \\
\text { Luteolin 6-fucoside } \\
\text { Luteolin 8-deoxyhexoside } \\
\text { Luteolin 6,8-diglucoside }\end{array}$ & HSS T3 & $0.1 \%$ FA: $\mathrm{H}_{2} \mathrm{O}(\mathrm{A}), 0.1 \% \mathrm{FA}: \mathrm{ACN}(\mathrm{B})$ & $\begin{array}{l}\text { injection volume: } 3.1 \mu \mathrm{L} \text {; } \\
\text { flow rate: } 0.15 \mathrm{~mL} / \mathrm{min}\end{array}$ & PDA-MS & Passiflora edulis & [128] \\
\hline $\begin{array}{l}\text { Luteolin 6,8-diglucoside } \\
\text { Luteolin 8-glucosyl-7-glucoside isomer } \\
\text { Luteolin 6-glucosyl-7-glucoside isomer }\end{array}$ & Kinetex $\mathrm{C}_{18}$ & $\begin{array}{l}0.05 \% \mathrm{FA}: \mathrm{H}_{2} \mathrm{O}(\mathrm{A}), 0.05 \% \mathrm{FA}: \mathrm{ACN} \\
\text { (B) }\end{array}$ & $\begin{array}{l}\text { flow rate: } 0.4 \mathrm{~mL} / \mathrm{min} \text {; } \\
\qquad 40^{\circ} \mathrm{C}\end{array}$ & MS/MS & Eragrostis tef & [84] \\
\hline \multicolumn{7}{|l|}{$\begin{array}{l}\text { Luteolin 8-glucosyl-7-rhamnoside } \\
\text { Luteolin 8-glucoside } \\
\text { Luteolin 7-glucoside } \\
\text { Luteolin 7-rhamnoside } \\
\text { Luteolin } \\
\text { 7-(6"-syringly)glucosyl-6-glucoside } \\
\text { Luteolin } \\
\text { 7-(2"-syringyl)arabinosyl-6-glucoside } \\
\text { Luteolin 8-(6"-diacetyl)glucoside }\end{array}$} \\
\hline $\begin{array}{c}\text { Luteolin } \\
\text { Luteolin 6-glucoside }\end{array}$ & Nucleosil 100-3.5 $C_{18}$ & $\mathrm{FA}: \mathrm{H}_{2} \mathrm{O}(\mathrm{A}), \mathrm{FA}: \mathrm{ACN}(\mathrm{B})$ & injection volume: $20 \mu \mathrm{L}$ & MS & Arum hygrophilum & [129] \\
\hline
\end{tabular}


Table 4. Cont.

\begin{tabular}{|c|c|c|c|c|c|c|}
\hline Luteolin Derivative & $\begin{array}{c}\text { Stationary } \\
\text { Phase/Column }\end{array}$ & Mobile Phase & Conditions & Detection & Analyzed Species & Ref. \\
\hline $\begin{array}{c}\text { Luteolin } \\
\text { 6-[6"-glocosy-caffeoyl-glucopyranosyl } \\
(" \rightarrow 2) \text {-glucopyranoside } \\
\text { Luteolin 6-glucopyranoside }\end{array}$ & Eclipse Plus $C_{18}$ & $0.2 \%$ FA: $\mathrm{H}_{2} \mathrm{O}(\mathrm{A}), 0.2 \%$ FA:ACN (B) & $30^{\circ} \mathrm{C}$ & $\begin{array}{l}\text { PDA-ESI-MS/MS } \\
\qquad \mathrm{UV}, 350 \mathrm{~nm}\end{array}$ & Triticum aestivum & [130] \\
\hline $\begin{array}{l}\text { Luteolin 7-glucoside } \\
\text { Luteolin 6-glucoside } \\
\text { Luteolin 7-(2"-p-coumaroyl)-rhamnoside }\end{array}$ & Syncronis $\mathrm{C}_{18}$ & $0.1 \% \mathrm{FA}: \mathrm{H}_{2} \mathrm{O}(\mathrm{A}), \mathrm{ACN}(\mathrm{B})$ & $\begin{array}{l}\text { injection volume: } 5 \mu \mathrm{L} \text {; } \\
\text { flow rate: } 0.25 \mathrm{~mL} / \mathrm{min}\end{array}$ & MS/MS & $\begin{array}{l}\text { Iris pumila, } \\
\text { I. variegata, } \\
\text { I. humilis }\end{array}$ & [131] \\
\hline $\begin{array}{c}\text { Luteolin } \\
\text { Luteolin hexoside }\end{array}$ & $\begin{array}{l}\text { Luna Omega Polar } \\
\mathrm{C}_{18} \text { with Polar } \mathrm{C}_{18}\end{array}$ & $0.1 \% \mathrm{FA}: \mathrm{H}_{2} \mathrm{O}(\mathrm{A}), \mathrm{ACN}(\mathrm{B})$ & flow rate: $0.4 \mathrm{~mL} / \mathrm{min}$ & $\begin{array}{l}\text { ESI-MS/MS; } \\
320,350 \mathrm{~nm}\end{array}$ & Parentucellia latifolia & {$[132,133]$} \\
\hline $\begin{array}{c}\text { Luteolin } \\
\text { Luteolin glucoside } \\
\text { Luteolin glucuronide } \\
\text { Luteolin pentosyl-glucoside }\end{array}$ & $\begin{array}{l}\text { Security Guard } \\
\text { cartridge } \\
\text { Intersil ODS }\end{array}$ & $\begin{array}{l}\text { ACN:H }{ }_{2} \mathrm{O}: \mathrm{FA}(10: 89: 1 v / v / v)(\mathrm{A}), \\
\text { ACN:H } \mathrm{H}_{2} \mathrm{O}: \mathrm{FA}(89: 10: 1 v / v / v)(\mathrm{B})\end{array}$ & $\begin{array}{l}\text { injection volume: } 10 \mu \mathrm{L} \text {; } \\
\text { flow rate: } 0.5 \mathrm{~mL} / \mathrm{min} \text {; } \\
40{ }^{\circ} \mathrm{C}\end{array}$ & $\begin{array}{l}\text { DAD-MS/MS; } \\
\quad 360 \mathrm{~nm}\end{array}$ & $\begin{array}{c}\text { Verbascum } \\
\text { eskisehirensis }\end{array}$ & [134] \\
\hline Luteolin $4^{\prime}$-glucoside & Kinetex RP $\mathrm{C}_{18}$ & $\mathrm{PA}: \mathrm{H}_{2} \mathrm{O} \mathrm{pH} 3(\mathrm{~A}), \mathrm{PA}: \mathrm{ACN} \mathrm{pH} 3$ (B) & $\begin{array}{l}\text { injection volume: } 1 \mu \mathrm{L} ; \\
\text { flow rate: } 1.2 \mathrm{~mL} / \mathrm{min} ; 35^{\circ} \mathrm{C}\end{array}$ & $\begin{array}{c}\mathrm{UV} \\
300 \mathrm{~nm}\end{array}$ & Matricaria recutita & [135] \\
\hline Luteolin 7-O-glucuronide & $\mathrm{C}_{18}$ & $\begin{array}{c}0.02 \% \text { TFA: } \mathrm{H}_{2} \mathrm{O}(\mathrm{A}), \\
\text { MeOH:ACN }(3: 7 v / v)(\mathrm{B})\end{array}$ & $\begin{array}{c}\text { injection volume: } 2.5 \mu \mathrm{L} ; \\
\text { flow rate: } 0.9 \mathrm{~mL} / \mathrm{min} ; 45^{\circ} \mathrm{C}\end{array}$ & $\begin{array}{c}\text { DAD-ESI-MS } \\
240,254,325 \mathrm{~nm}\end{array}$ & Lippia alba & [136] \\
\hline $\begin{array}{c}\text { Luteolin glucoside } \\
\text { Luteolin 7-O- } \beta \text {-glucoside } \\
\text { Luteolin glucuronide } \\
\text { Luteolin malonylglucoside } \\
\text { Luteolin }\end{array}$ & YMC-Triart $C_{18}$ & $0.1 \%$ FA: $\mathrm{H}_{2} \mathrm{O}(\mathrm{A}), 0.1 \%$ FA:ACN B) & $\begin{array}{l}\text { injection volume: } 10 \mu \mathrm{L} \text {; } \\
\text { flow rate: } 0.8 \mathrm{~mL} / \mathrm{min}\end{array}$ & $\begin{array}{c}\text { DAD-ESI-MA } \\
265,280,330 \text {, and } 360 \mathrm{~nm}\end{array}$ & $\begin{array}{l}\text { Chrysanthemum } \\
\text { morifolium }\end{array}$ & [137] \\
\hline $\begin{array}{l}\text { Luteolin 7-diglucuronide } \\
\text { Luteolin 7-O-glucuronide }\end{array}$ & Eclipse XDB $C_{18}$ & $\begin{array}{c}0.03 \% \text { PA: } \mathrm{H}_{2} \mathrm{O}(\mathrm{A}) \\
\text { solvent A:ACN }(1: 9 \mathrm{v} / \mathrm{v})(\mathrm{B})\end{array}$ & $\begin{array}{l}\text { injection volume: } 20 \mu \mathrm{L} \text {; } \\
\text { flow rate: } 0.8 \mathrm{~mL} / \mathrm{min} \text {; }\end{array}$ & $\begin{array}{c}\text { DAD, 210, 250, } 320,350 \text { and } \\
370 \mathrm{~nm}\end{array}$ & Thymus pannonicus & [138] \\
\hline
\end{tabular}

Abbreviations: Ace, acetone; $\mathrm{AcOH}$, acetic acid; $\mathrm{ACN}$, acetonitrile; $\mathrm{AFNH}_{4}$, ammonium formate; $\mathrm{BuOH}$, butanol; DAD, diode array detector; DAD-ESI-MS, diode array detector combined with electrospray ionization and mass spectrometry; DAD-ESI-MS/MS, diode array detector combined with electrospray ionization and tandem mass spectrometry; DAD-ESI-QTOF-MS, diode array detector combined with electrospray ionization and quadrupole - time of flight mass spectrometry; DAD-MS, diode array detector combined with mass spectrometry; DAD-MS/MS, diode array detector combined with tandem mass spectrometry; DAD-QTOF-MS, diode array detector combined with quadrupole - time of flight mass spectrometry; ESI-IT-MS/MS, electrospray ionization combined with ion trap and tandem mass spectrometry; ESI-MS, electrospray ionization combined with mass spectrometry; ESI-MS/MS, electrospray ionization combined with tandem mass spectrometry; ESI-OTOF-MS, electrospray ionization combined with quadrupole - time of flight mass spectrometry; FA, formic acid; IMS-QTOF-MS, ion-mobility spectrometry combined with quadrupole - time of flight mass spectrometry; $\mathrm{MeOH}$, methanol; MS/MS, tandem mass spectrometry; PA, phosphoric acid; PAD, pulsed amperometric detection; PDA-ESI-MS/MS, pulsed amperometric detection combined with electrospray ionization and tandem mass spectrometry; PDA-ESI-MS, pulsed amperometric detection combined with electrospray ionization and mass spectrometry; PDA-MS, pulsed amperometric detection combined with mass spectrometry; TFA, trifluoroacetic acid; UV/PAD-MS, UV/pulsed amperometric detection combined with mass spectrometry. 


\subsection{Liquid Chromatography in the Analysis of Luteolin Derivatives}

LC combined with tandem mass spectrometry (LC-MS/MS) (Table 5) is especially useful in the analysis of multicomponent mixtures, such as herbal extracts because it does not require a large amount of the sample or previous separation. To further reduce the influence of other factors on the analysis, more advanced techniques, involving the combination of more than one detection method, e.g., LC combined with NMR and MS (LC-NMR-MS), are increasingly used [139].

Research on ethanol extract from Lophatherum gracile stems and leaves was performed using LC coupled with MS/MS. Gradient elution was performed using $0.3 \% \mathrm{FA}$ and $\mathrm{MeOH}$. Analysis of the species revealed the presence of, among others, luteolin 7-O- $\beta$-D-glucoside, and luteolin 6-C-glucoside [140].

Another raw material containing luteolin and its derivatives is the cocoa seed (Theobroma cacao). In the analysis of the $\mathrm{H}_{2} \mathrm{O}: \mathrm{MeOH}$ extract, the following mobile phase was used: $\mathrm{H}_{2} \mathrm{O}: 0.1 \% \mathrm{FA}$ and ACN:0.1\% FA. The elution was carried out in a linear gradient, whereas LC combined with electrospray ionization and tandem mass spectrometry (LC-ESI-MS/MS) coupling allowed the identification of the tested compounds [141].

Lin and Harnly performed a water-methanol analysis of the flower extract of Chrysanthemum morifolium and distinguished many compounds, including numerous derivatives of luteolin. In this case, the mobile phase was a mixture of $0.1 \% \mathrm{FA}: \mathrm{H}_{2} \mathrm{O}$ and $0.1 \% \mathrm{FA}: \mathrm{ACN}$, in varying proportions. The qualitative determination of the analyzed substances was based on a comparison of retention times as well as mass and UV/Vis spectra [142]. 
Table 5. Liquid chromatography in the analysis of luteolin derivatives.

\begin{tabular}{|c|c|c|c|c|c|c|}
\hline Luteolin Derivative & Stationary Phase & Mobile Phase & Conditions & Detection & Analyzed Species & Ref. \\
\hline \multirow{3}{*}{ Luteolin } & $\begin{array}{c}\text { Zorbax } \mathrm{SB} \mathrm{C}_{18} \\
\text { column with } \\
\text { Security-Guard } \mathrm{C}_{18}\end{array}$ & $\mathrm{MeOH}(\mathrm{A}), 0.5 \% \mathrm{AcOH}: \mathrm{H}_{2} \mathrm{O}(\mathrm{B})$ & $\begin{array}{l}\text { flow rate: } 1.0 \mathrm{~mL} / \mathrm{min} \text {; } \\
\text { injection volume: } 20 \mu \mathrm{L}\end{array}$ & MS/MS & $\begin{array}{l}\text { Abri herba, } \\
\text { A. mollis }\end{array}$ & [143] \\
\hline & Inertsil ODS-3 & $0.1 \% \mathrm{FA}: \mathrm{H}_{2} \mathrm{O}(\mathrm{A}), \mathrm{ACN}(\mathrm{B})$ & $\begin{array}{l}\text { flow rate: } 0.5 \mathrm{~mL} / \mathrm{min} \text {; } \\
\text { injection volume: } 10 \mu \mathrm{L}\end{array}$ & MS/MS & Castanea mollissima & [144] \\
\hline & $\mathrm{RPC}_{18}$ & $0.1 \% \mathrm{FA}: \mathrm{H}_{2} \mathrm{O}(\mathrm{A}), 0.1 \%$ FA:ACN (B) & $\begin{array}{c}\text { injection volume: } 5 \mu \mathrm{L} \text {; } \\
\text { flow rate: } 0.3 \mathrm{~mL} / \mathrm{min} \text {; } \\
40^{\circ} \mathrm{C}\end{array}$ & ESI-MS/MS & Centaurea cyanus & [145] \\
\hline Luteolin 7-glucoside & Eclipse XDB $C_{18}$ & $1 \%$ FA: $\mathrm{H}_{2} \mathrm{O}(\mathrm{A}), \mathrm{MeOH}(\mathrm{B})$ & $\begin{array}{c}\text { injection volume: } 5 \mathrm{~mL} \text {; } \\
\text { flow rate: } 0.6 \mathrm{~mL} / \mathrm{min} \text {; } \\
45^{\circ} \mathrm{C}\end{array}$ & MS/MS & $\begin{array}{c}\text { Plantago atrata, } \\
\text { P. coronopus, } \\
\text { P. holosteum, } \\
\text { P. lanceolata, } \\
\text { P. reniformis, } \\
\text { P. schwarzenbergiana }\end{array}$ & [146] \\
\hline \multirow[t]{2}{*}{ Luteolin } & Zorbax Plus $\mathrm{C}_{18}$ & $0.1 \% \mathrm{FA}: \mathrm{H}_{2} \mathrm{O}(\mathrm{A}), 0.1 \% \mathrm{FA}: \mathrm{ACN}(\mathrm{B})$ & $\begin{array}{l}\text { injection volume: } 1 \mu \mathrm{L} \text {; } \\
\text { flow rate: } 0.4 \mathrm{~mL} / \mathrm{min}\end{array}$ & MS & $\begin{array}{l}\text { Matricaria recutita, } \\
\text { Achillea millefolium, } \\
\text { Thymus vulgaris, } \\
\text { Salvia officinalis } \\
\end{array}$ & {$[30]$} \\
\hline & Eclipse XDB C 18 & $0.05 \%$ FA: $\mathrm{H}_{2} \mathrm{O}(\mathrm{A}), \mathrm{MeOH}(\mathrm{B})$ & flow rate: $1 \mathrm{~mL} / \mathrm{min}$ & MS/MS & Vitis vinifera & [147] \\
\hline $\begin{array}{l}\text { Luteolin 7-apiosyl-glucoside } \\
\text { Luteolin 7-glucoside } \\
\text { Luteolin } \\
\text { 7-malonyl-apiosyl-glucoside } \\
\text { Luteolin } \\
\text { 7-6'-malonyl-apiosyl-glucoside } \\
\text { Luteolin } \\
\text { 7-6'-malonyl-glucoside }\end{array}$ & Symmetry $C_{18}$ & $0.1 \%$ FA: $\mathrm{H}_{2} \mathrm{O}(\mathrm{A}), 0.1 \%$ FA:ACN (B) & $\begin{array}{l}\text { flow rate: } 1.0 \mathrm{~mL} / \mathrm{min} ; \\
\qquad 25^{\circ} \mathrm{C}\end{array}$ & $\begin{array}{c}\text { DAD-ESI-MS; } \\
\text { DAD, } 350,310,270 \mathrm{~nm} ; \\
\text { UV, } 190-650 \mathrm{~nm}\end{array}$ & Apium graveolens & [148] \\
\hline $\begin{array}{l}\text { Luteolin 3'-glucoside } \\
\text { Luteolin 6-glucoside } \\
\text { Luteolin 6,8-dihexoside } \\
\text { Luteolin 7-rutinoside }\end{array}$ & Zorbax SB $\mathrm{C}_{18}$ & $0.1 \%$ FA: $\mathrm{H}_{2} \mathrm{O}(\mathrm{A}) ; 0.1 \%$ FA:ACN (B) & $\begin{array}{l}\text { injection volume: } 5 \mu \mathrm{L} \text {; } \\
\text { flow rate: } 0.3 \mathrm{~mL} / \mathrm{min} ; \\
25^{\circ} \mathrm{C}\end{array}$ & MS/MS; TQMS; ESI & $\begin{array}{c}\text { Phlomis persica } \\
\text { Ph. eliptica }\end{array}$ & {$[27]$} \\
\hline $\begin{array}{c}\text { Luteolin 7-rutinoside } \\
\text { Luteolin 7-glucopyranoside }\end{array}$ & XTerra MS $C_{18}$ & $\mathrm{ACN}(\mathrm{A}), 0.05 \% \mathrm{AcOH}: \mathrm{H}_{2} \mathrm{O}(\mathrm{B})$ & $\begin{array}{l}\text { injection volume: } 20 \mu \mathrm{L} ; \\
\text { flow rate: } 1.0 \mathrm{~mL} / \mathrm{min}\end{array}$ & PDA-MS; NMR & Sechium edule & [149] \\
\hline
\end{tabular}


Table 5. Cont.

\begin{tabular}{|c|c|c|c|c|c|c|}
\hline Luteolin Derivative & Stationary Phase & Mobile Phase & Conditions & Detection & Analyzed Species & Ref. \\
\hline $\begin{array}{c}\text { Luteolin } \\
\text { Luteolin glucuronide } \\
\text { Luteolin glucoside }\end{array}$ & $\begin{array}{l}\text { Octadecyl silica gel } \\
\text { ODS }\end{array}$ & $\begin{array}{l}\mathrm{H}_{2} \mathrm{O}: \mathrm{MeOH}: \mathrm{FA}(89: 10: 1 v / v / v)(\mathrm{A}) \\
\text { MeOH:H } \mathrm{H}_{2} \mathrm{O}: \mathrm{FA}(89: 10: 1 v / v / v)(\mathrm{B})\end{array}$ & $\begin{array}{l}\text { flow rate: } 1 \mathrm{~mL} / \mathrm{min} ; \\
40^{\circ} \mathrm{C}\end{array}$ & MS/MS & Nepeta cilicica & [150] \\
\hline $\begin{array}{c}\text { Luteolin } \\
\text { Luteolin dihexoside } \\
\text { Luteolin 7-dihexoside } \\
\text { Luteolin 7-rutinoside } \\
\text { Luteolin glucoside } \\
\text { Luteolin 7-acetylglucoside }\end{array}$ & Capcell Park $\mathrm{C}_{18}$ & $0.5 \%$ FA: $\mathrm{H}_{2} \mathrm{O}(\mathrm{A}), 0.5 \%$ FA:ACN (B) & $\begin{array}{l}\text { flow rate: } 0.5 \mathrm{~mL} / \mathrm{min} \text {; } \\
\qquad 25^{\circ} \mathrm{C}\end{array}$ & MS/MS & Humulus japonicus & [151] \\
\hline $\begin{array}{l}\text { Luteolin 6-glucoside } \\
\text { Luteolin glucoside }\end{array}$ & Intersil ODS & $\begin{array}{l}\text { ACN: } \mathrm{H}_{2} \mathrm{O}: F A(10: 89: 1 v / v / v)(\mathrm{A}) \\
\text { ACN:H } \\
2\end{array}$ & $\begin{array}{l}\text { flow rate: } 0.7 \mathrm{~mL} / \mathrm{min} \text {; } \\
40^{\circ} \mathrm{C}\end{array}$ & MS/MS & Achillea sivasica & [152] \\
\hline \begin{tabular}{l}
\multicolumn{1}{c}{ Luteolin } \\
methoxy-2"-pentosyl-6-hexoside \\
$\quad$ Luteolin \\
2"-rhamnosyl-6"-hexosyl-glucoside \\
Luteolin 7-glucosyl-8-glucoside \\
$\quad$ Luteolin 6-glucoside \\
Luteolin 6-hexosyl-8-pentoside
\end{tabular} & ODS2 $C_{8}$ & $0.1 \% \mathrm{FA}: \mathrm{H}_{2} \mathrm{O}(\mathrm{A}), \mathrm{ACN}(\mathrm{B})$ & $\begin{array}{l}\text { flow rate: } 0.5 \mathrm{~mL} / \mathrm{min} \text {; } \\
\qquad 35^{\circ} \mathrm{C}\end{array}$ & $\begin{array}{l}\text { DAD-ESI-MS; } \\
\text { 280, } 370 \mathrm{~nm}\end{array}$ & Geranium molle & {$[68,70,153]$} \\
\hline $\begin{array}{l}\text { Luteolin acetylhexoside } \\
\text { Luteolin 6-glucoside } \\
\text { Luteolin 3-glucuronide } \\
\text { Luteolin glucuronide }\end{array}$ & $\begin{array}{l}\text { Spherisorb S3 ODS2 } \\
\qquad \mathrm{C}_{18}\end{array}$ & $0.1 \% \mathrm{FA}: \mathrm{H}_{2} \mathrm{O}(\mathrm{A}), \mathrm{ACN}(\mathrm{B})$ & $\begin{array}{l}\text { flow rate: } 0.5 \mathrm{~mL} / \mathrm{min} \text {; } \\
\qquad 35^{\circ} \mathrm{C}\end{array}$ & DAD-ESI-MS/MS & $\begin{array}{l}\text { Achillea millefolium } \\
\text { Coleostephus myconis } \\
\text { Rosmarinus officinalis }\end{array}$ & $\begin{array}{c}{[120,154]} \\
{[120]} \\
{[155]}\end{array}$ \\
\hline $\begin{array}{l}\text { Luteolin hexosyl-pentoside } \\
\text { Luteolin 7-glucoside } \\
\text { Luteolin 7-hexoside } \\
\text { Luteolin 6-hexoside } \\
\text { Luteolin 8-glucoside }\end{array}$ & Zorbax $\mathrm{RP}_{18}$ & $0.1 \%$ FA: $\mathrm{H}_{2} \mathrm{O}(\mathrm{A}), 0.1 \%$ FA:ACN (B) & $\begin{array}{l}\text { injection volume: } 5 \mu \mathrm{L} \text {; } \\
\text { flow rate: } 0.4 \mathrm{~mL} / \mathrm{min} \text {; } \\
\qquad 35^{\circ} \mathrm{C}\end{array}$ & QTOF & Ficus carica & [156] \\
\hline $\begin{array}{c}\text { Luteolin } \\
\text { 6-hexosyl-8-acetyl-hexoside } \\
\text { Luteolin } \\
\text { 8-glucosyl-7,3'-dimethoxyl- } \\
\text { 2"-O-glycoside } \\
\text { Luteolin } \\
\text { 8-glucosyl-7,3'-dimethoxyl- } \\
\text { 6-desoxyhexoside }\end{array}$ & ODS2 & $\mathrm{H}_{2} \mathrm{O}: \mathrm{ACN}: \mathrm{FA}$ & $\begin{array}{l}\text { injection volume: } 20 \mu \mathrm{L} \text {; } \\
\text { flow rate: } 0.8 \mathrm{~mL} / \mathrm{min}\end{array}$ & MS/MS & Oxalis pes-caprae & [157] \\
\hline
\end{tabular}


Table 5. Cont.

\begin{tabular}{|c|c|c|c|c|c|c|}
\hline Luteolin Derivative & Stationary Phase & Mobile Phase & Conditions & Detection & Analyzed Species & Ref. \\
\hline $\begin{array}{l}\text { Luteolin 6-glucoside } \\
\text { Luteolin 8-glucoside }\end{array}$ & Hydro RP & $\begin{array}{l}\mathrm{H}_{2} \mathrm{O}(\mathrm{A}), \mathrm{MeOH}(\mathrm{B}), \\
5 \% \text { AcOH:MeOH (C) }\end{array}$ & flow rate: $1 \mathrm{~mL} / \mathrm{min}$ & MS/MS & Passiflora morifolia & [158] \\
\hline $\begin{array}{l}\text { Luteolin } \\
\text { Luteolin 6-glucoside } \\
\text { Luteolin 8-glucoside } \\
\text { Luteolin 7-glucoside }\end{array}$ & Luna $\mathrm{C}_{18}$ & $0.1 \% \mathrm{FA}: \mathrm{H}_{2} \mathrm{O}(\mathrm{A}), 0.1 \%$ FA:ACN (B) & flow rate: $0.4 \mathrm{~mL} / \mathrm{min}$ & $\begin{array}{l}\text { DAD, 280, 320, } 365 \mathrm{~nm} ; \\
\text { MS; MS/MS }\end{array}$ & Theobroma cacao & [141] \\
\hline $\begin{array}{l}\text { Luteolin 6-glucosie } \\
\text { Luteolin 7-glucoside }\end{array}$ & XBridge $\mathrm{C}_{18}$ & $0.3 \% \mathrm{FA}: \mathrm{H}_{2} \mathrm{O}(\mathrm{A}), \mathrm{MeOH}(\mathrm{B})$ & $\begin{array}{l}\text { injection volume: } 1 \mu \mathrm{L} \text {; } \\
\text { flow rate: } 1 \mathrm{~mL} / \mathrm{min} \text {; }\end{array}$ & MS/MS; ESI-MS & Lophatherum gracile & [140] \\
\hline $\begin{array}{c}\text { Luteolin } \\
\text { Luteolin glucuronyl-hexoside } \\
\text { Luteolin 7-pentosyl-hexoside } \\
\text { Luteolin 7-rutinoside } \\
\text { Luteolin 7-glucoside } \\
\text { Luteolin 7-glucuronide } \\
\text { Luteolin glucoside } \\
\text { Luteolin } \\
\text { 7-malonyl-6"-glucoside } \\
\text { Luteolin 7-acetyl-6"-glucoside } \\
\text { Luteolin 7-dihexoside }\end{array}$ & Symmetry $C_{18}$ & $0.1 \%$ FA: $\mathrm{H}_{2} \mathrm{O}(\mathrm{A}), 0.1 \%$ FA:ACN (B) & $\begin{array}{l}\text { flow rate: } 1.0 \mathrm{~mL} / \mathrm{min} ; \\
225^{\circ} \mathrm{C}\end{array}$ & $\begin{array}{l}\text { DAD-ESI-MS; } \\
350,310,270 \\
\text { and } 520 \mathrm{~nm}\end{array}$ & $\begin{array}{l}\text { Chrysanthemum } \\
\text { morifolium }\end{array}$ & [142] \\
\hline $\begin{array}{c}\text { Luteolin } \\
\text { Luteolin7-glucuronide }\end{array}$ & Acquity $\mathrm{BEH} \mathrm{C}_{18}$ & $0.1 \%$ FA: $\mathrm{H}_{2} \mathrm{O}(\mathrm{A}), 0.1 \%$ FA:ACN (B) & $\begin{array}{l}\text { flow rate: } 0.3 \mathrm{~mL} / \mathrm{min} \text {; } \\
\qquad 40^{\circ} \mathrm{C}\end{array}$ & QTOF-MS/MS & Ageratum conyoides & [159] \\
\hline Luteolin hexoside & Zorbax $\mathrm{SB} \mathrm{C}_{18}$ & $0.5 \% \mathrm{FA}: \mathrm{H}_{2} \mathrm{O}(\mathrm{A}), \mathrm{ACN}(\mathrm{B})$ & $\begin{array}{l}\text { injection volume: } 5 \mu \mathrm{L} \text {; } \\
\text { flow rate: } 0.4 \mathrm{~mL} / \mathrm{min}\end{array}$ & MS/MS & Matricaria recutita & [160] \\
\hline $\begin{array}{l}\text { Luteolin hexoside } \\
\text { Luteolin hexosyl-deoxy-hexose }\end{array}$ & Hypersil gold $C_{18}$ & $1 \%$ FA: $\mathrm{H}_{2} \mathrm{O}(\mathrm{A}), 0.1 \%$ FA:ACN (B) & flow rate: $1 \mathrm{~mL} / \mathrm{min}$ & MS/MS & Cecropia obtusa & [161] \\
\hline $\begin{array}{c}\text { Luteolin dihexoside } \\
\text { Luteolin pentosyl-hexoside } \\
\text { Luteolin 7-glucoside } \\
\text { Luteolin malonyl-hexoside }\end{array}$ & $\begin{array}{c}\text { Spherisorb S3 ODS-2 } \\
\mathrm{C}_{18}\end{array}$ & $\begin{array}{l}0.1 \% \text { FA: } \mathrm{H}_{2} \mathrm{O}(\mathrm{A}) \\
\text { ACN (B) }\end{array}$ & $\begin{array}{l}\text { flow rate: } 0.5 \mathrm{~mL} / \mathrm{min} ; \\
35^{\circ} \mathrm{C} .\end{array}$ & DAD-ESI-MS/MS & Cotula cinerea & {$[120,162]$} \\
\hline $\begin{array}{c}\text { Luteolin } \\
\text { Luteolin 8-glucoside } \\
\text { Luteolin 5-glucopyranoside }\end{array}$ & Zorbax $\mathrm{SB} \mathrm{C}_{18}$ & $\begin{array}{c}0.1 \% \mathrm{FA}^{\mathrm{AFNH}} \mathrm{AF}_{4}(\mathrm{~A}), \\
0.1 \% \mathrm{FA}: \mathrm{ACN}(\mathrm{B})\end{array}$ & $\begin{array}{l}\text { injection volume: } 1 \mathrm{~mL} \text {; } \\
\text { flow rate: } 0.2 \mathrm{~mL} / \mathrm{min}\end{array}$ & ESI-MS/MS & Ocimum sanctum & [163] \\
\hline
\end{tabular}

Abbreviations: $\mathrm{AcOH}$, acetic acid; $\mathrm{ACN}$, acetonitrile; $\mathrm{AFNH}_{4}$, ammonium formate; DAD-ESI-MS, diode array detector combined with electrospray ionization and mass spectrometry; DAD, diode array detector; DAD-ESI-MS/MS, diode array detector combined with electrospray ionization and tandem mass spectrometry; ESI, electrospray ionization; ESI-MS, electrospray ionization combined with mass spectrometry; FA, formic acid; MeOH, methanol; MS/MS, tandem mass spectrometry; PDA-MS, pulsed amperometric detection combined with mass spectrometry; QTOF, quadrupole time-of-flight; TQMS, triple quadrupole mass spectrometer. 


\subsection{Gas Chromatography in the Analysis of Luteolin and Its Derivatives}

Gas chromatography (GC) is a technique used to analyze volatile as well as non-volatile compounds after their derivatization. GC is characterized by chromatographic distribution of either a gas mobile phase on a solid adsorbent (gas-solid chromatography) or a liquid on an inert support (gas-liquid chromatography). GC can be hyphenated with various detection techniques such as GC combined with mass spectrometry (GC-MS), GC combined with tandem mass spectrometry (GC-MS/MS) or GC combined with time of flight mass spectrometry (GC-TOF-MS), thus greatly increasing the versatility, sensitivity and accuracy of the method $[164,165]$. The analyzed substances should be thermally stable, and their boiling (or sublimation) temperature should not exceed $350-400^{\circ} \mathrm{C}$. To achieve this, non-volatile substances are often derivatived. Polar functional groups are transformed into their less polar counterparts, thus increasing the volatility of the prepared compounds. The most common examples include substitution with a trimethylsilyl (TM) group, organic radicals or compounds such as trimethylchlorosilane (TMCS), hexamethyldisilazane (HMDS) or N,O-bis(trimethylsilyl)-trifluoroacetamide (BSTFA). Such an approach greatly increases the number of possible analytes [2,17]. For example, phenolic groups of flavonoids are often transformed into their less polar trimethylsilyl counterparts allowing for rapid and effective separation of complex mixtures [166].

In adsorption GC, a gas that is chemically inert to the stationary phase as well as the components being analyzed is used as the mobile phase. Most often hydrogen, nitrogen or argon is used. Helium is being used less often due to its higher cost than other gases and the implementation of the principles of chemical safety. The mobile phase must be properly selected for compatibility with the detector used. However, the carrier gas itself does not have a significant influence on the separation effects of the analyzed mixtures [17]. In the process of separation, the method of application of the sample to the chromatography column is very important. The sample should always have as small of a volume and the shortest dosing time as possible. This ensures better separation and narrower bands $[17,164]$.

Gas chromatography uses open-ended columns, i.e., capillary columns and packed columns. Open-ended (OT-open tubular) columns are characterized by much higher efficiency than packed columns; therefore, they are chosen much more often [164]. Capillary columns are particularly useful in the separation of substances with significantly different boiling points. Ideally, the column should have a similar polarity to the analyzed components. However, due to the higher efficiency and greater durability of stationary phases with low polarity, so capillary columns are recommended for chromatographic analyses [17,164].

Another factor that influences the efficiency of the separation of the analyzed sample components, as well as the time of analysis, is temperature. The separation temperature should be selected depending on the stationary phase used and the boiling point of the analytes [17,164].

The analysis of luteolin derivatives has been carried out in accordance with the general procedure used for analysis of other flavonoids (Table 6) [165]. MS was used for detection of volatile derivatives of luteolin, while the use of a flame ionization detector (FID) was described only in two papers $[167,168]$. Most commonly, helium was used as a carrier gas (e.g., [169-171]), but the use of nitrogen was also recorded $[167,168]$. The derivatization of luteolin, which is necessary for chromatographic separation, was mostly achieved with BSTFA/TCMS [170-172]. For the analysis of lipophilic luteolin derivatives, such as 7,3', $4^{\prime}$-trimethyl-luteolin in Arnica alpina, no derivatization was necessary [168].

\subsection{Counter-Current Chromatography in the Analysis of Luteolin Derivatives}

Counter-current chromatography (CCC) is a variation of liquid chromatography in which both the stationary and mobile phases are liquid. The separation of the constituents of the mixture is carried out in a system of immiscible liquids that are in equilibrium with each other. The method is simple and rapid, offering the possibility of introducing the raw sample to the column without need for previous clean-up [173]. Counter-current chromatography is mainly used for purification of natural compounds, while its use as an analytical technique is far less common. In CCC, various dividing 
techniques can be used, thus distinguishing centrifugal partition chromatography (CPC) and rapid- or high-speed CCC (HSCCC), often referred to as hydrodynamic chromatography [174,175]. High-speed CCC is particularly often encountered in studies involving the separation of flavonoids [176]. Table 7 presents the conditions for the separation of mixtures containing luteolin and its derivatives using rapid counter-current chromatography. Separation of luteolin derivatives from mixtures of different phytochemicals is usually carried out with mixtures of EtOAc with one of alcohol $(\mathrm{BuOH}, \mathrm{EtOH}$ or $\mathrm{AcOH}$ ) and $\mathrm{H}_{2} \mathrm{O}$. Upon separation, structure determination by NMR is often necessary $[173,177]$. However, instead of NMR, high resolution mass spectrometry (HRMS) can also be employed for structure confirmation, as evidenced by the use of this technique for differentiation of various luteolin derivatives in Lippia origanoides [178]. 
Table 6. Gas chromatography in the analysis of luteolin and its derivatives.

\begin{tabular}{|c|c|c|c|c|c|c|}
\hline Luteolin Derivative & Column & Derivatization & Conditions & Detection & Analyzed Species & Ref. \\
\hline \multirow{6}{*}{ Luteolin } & HP-5-MS & 1\% TMCS:BSTFA & $\begin{array}{c}\text { carrier gas: } \mathrm{He} ; \\
\text { injector temperature: } 250^{\circ} \mathrm{C} \\
\text { A: (column temperature) } 5 \mathrm{~min}, 170^{\circ} \mathrm{C} ; \\
3{ }^{\circ} \mathrm{C} / \mathrm{min}, 170-255^{\circ} \mathrm{C} ; 1 \mathrm{~min}, 255^{\circ} \mathrm{C} ; 2^{\circ} \mathrm{C} / \mathrm{min}, 255-310^{\circ} \mathrm{C} ; \\
\text { flow rate: } 0.5 \mathrm{~mL} / \mathrm{min} ; \\
\text { analysis time: } 70 \mathrm{~min} ; \\
\text { B: (column temperature) } 5 \mathrm{~min}, 160^{\circ} \mathrm{C} ; 3^{\circ} \mathrm{C} / \mathrm{min}, 160-188^{\circ} \mathrm{C} ; 1 \mathrm{~min}, 188 \\
{ }^{\circ} \mathrm{C} ; 15^{\circ} \mathrm{C} / \mathrm{min}, \\
188-241^{\circ} \mathrm{C} ; 1 \mathrm{~min}, 241^{\circ} \mathrm{C} ; 2^{\circ} \mathrm{C} / \mathrm{min}, \\
241-282{ }^{\circ} \mathrm{C} ; 5^{\circ} \mathrm{C} / \mathrm{min}, 282-310^{\circ} \mathrm{C} ; 5 \mathrm{~min}, \\
310^{\circ} \mathrm{C} ; \\
\text { flow rate: } 1.0 \mathrm{~mL} / \mathrm{min} ; \\
\text { analysis time: } 50 \mathrm{~min}\end{array}$ & APCI-TOF-MS & $\begin{array}{l}\text { Fruits of various olives species } \\
\text { (Olea } \mathrm{L} .)\end{array}$ & [170] \\
\hline & $\begin{array}{l}\text { Capillary column } \\
\text { Supelco SPBM-5 }\end{array}$ & $\begin{array}{l}\text { two- and three-phase } \\
\text { transfer catalysis } \\
\text { (PTC), methyl iodide }\end{array}$ & $\begin{array}{c}\text { carrier gas: } \mathrm{He} ; \\
\text { injector temperature: } 260^{\circ} \mathrm{C} ; \\
\text { detector temperature: } 280^{\circ} \mathrm{C} ; \\
\text { furnace temperature: } 5 \mathrm{~min}, 50^{\circ} \mathrm{C} ; 5^{\circ} \mathrm{C} / \mathrm{min}, 50-1500^{\circ} \mathrm{C} ; 10^{\circ} \mathrm{C} / \mathrm{min} \text {, } \\
\text { analysis time: } 45 \mathrm{~min}\end{array}$ & MS & $\begin{array}{l}\text { Mentha spicata, } \\
\text { Hypericum perforatum }\end{array}$ & [179] \\
\hline & non-polar RSL 200 BP & $\begin{array}{l}0.2 \mathrm{M} \text { trimethylaniline } \\
\text { hydroxide } \\
\text { (TMAH): } \mathrm{H}_{2} \mathrm{O} \text {-free } \\
\text { MeOH } \\
\text { Py:HMDS:TMCS }\end{array}$ & $\begin{array}{c}\text { carrier gas: } \mathrm{N}_{2} ; \\
\text { linear speed of the carrier } 17.5 \mathrm{~cm} / \mathrm{s} ; \\
0-2 \mathrm{~min}, 280^{\circ} \mathrm{C} \text { and } 235^{\circ} \mathrm{C} ; 1^{\circ} \mathrm{C} / \mathrm{min}, \\
280-290{ }^{\circ} \mathrm{C} ; \\
\text { flow rate: } 30 \mathrm{~mL} / \mathrm{min}\end{array}$ & FID & $\begin{array}{l}\text { different samples form AFRC } \\
\text { Institute of Plant Science Research } \\
\text { and John Innes Institute, Norwich, } \\
\text { U.K. }\end{array}$ & [167] \\
\hline & BPX5 & $\begin{array}{l}\text { Py:BSTFA:TMCS) } \\
\quad(50: 50: 1 v / v / v)\end{array}$ & $\begin{array}{c}\text { carrier gas: He; } \\
\text { injector temperature: } 310^{\circ} \mathrm{C} ; \\
1 \mathrm{~min}, 100^{\circ} \mathrm{C} ; 30^{\circ} \mathrm{C} / \mathrm{min}, 100-210^{\circ} \mathrm{C} ; 2^{\circ} \mathrm{C} / \mathrm{min}, 210-240{ }^{\circ} \mathrm{C} ; 4^{\circ} \mathrm{C} / \mathrm{min}, \\
240-270^{\circ} \mathrm{C} ; 5^{\circ} \mathrm{C} / \mathrm{min}, 270-310^{\circ} \mathrm{C} ; 5 \mathrm{~min}, 310^{\circ} \mathrm{C} ; \\
\text { flow rate: } 1.5 \mathrm{~mL} / \mathrm{min}\end{array}$ & QMS & $\begin{array}{c}\text { Propolis, } \\
\text { Chrysanthemum sp, } \\
\text { Theobroma cacao (bitter chocolate) }\end{array}$ & [171] \\
\hline & $\begin{array}{l}\text { Capillary column } \\
\text { Low-bleed CP-Sil } 8 \\
\quad \text { CB-MS }\end{array}$ & $\begin{array}{c}\text { TMCS }(100 \mu \mathrm{L}) \\
\text { BSTFA }(200 \mu \mathrm{L}) \\
\text { HMDS:TMCS:Py } \\
(3: 1: 9, v / v / v)\end{array}$ & $\begin{array}{c}\text { carrier gas: He; } \\
2{ }^{\circ} \mathrm{C} / \mathrm{min}, 70-135^{\circ} \mathrm{C} ; 10 \mathrm{~min}, 135^{\circ} \mathrm{C} ; 4^{\circ} \mathrm{C} / \mathrm{min}, 135-220^{\circ} \mathrm{C} ; 10 \mathrm{~min}, 220 \\
{ }^{\circ} \mathrm{C} ; 3.5^{\circ} \mathrm{C} / \mathrm{min}, 220-270^{\circ} \mathrm{C} ; 20 \mathrm{~min}, 270^{\circ} \mathrm{C} ; \\
\text { injector temperature: } 280^{\circ} \mathrm{C} ; \\
\text { detector temperature: } 2900^{\circ} \mathrm{C} ; \\
\text { flow rate: } 1.9 \mathrm{~mL} / \mathrm{min}\end{array}$ & MS & Teucrium polium & [169] \\
\hline & $\begin{array}{l}\text { Quartz capillary } \\
\text { column }\end{array}$ & $\begin{array}{l}\text { Py:BSTFA } \\
(1: 1 v / v)\end{array}$ & $\begin{array}{l}\text { carrier gas: He;injector temperature } 220^{\circ} \mathrm{C} \text {; } \\
\text { detector temperature: } 270{ }^{\circ} \mathrm{C} \text {; } \\
2.3^{\circ} \mathrm{C} / \text { min } 200-270{ }^{\circ} \mathrm{C} ; 30 \mathrm{~min}, 270^{\circ} \mathrm{C}\end{array}$ & MS & Aspalathus linearis & [172] \\
\hline
\end{tabular}


Table 6. Cont.

\begin{tabular}{ccccc}
\hline Luteolin Derivative & Column & Derivatization & Conditions & Detection \\
\hline Luteolin & Capillary column & - & carrier gas: $\mathrm{N}_{2}, \mathrm{He} ;$ & Analyzed Species \\
$7,3^{\prime} 4^{\prime}$-trimethyl & $\begin{array}{c}\text { Permabond OV-1 } \\
\text { Capillary column }\end{array}$ & - & injector temperature: $30{ }^{\circ} \mathrm{C} ;$ & FID \\
OV-1 & - & column temperature: $270{ }^{\circ} \mathrm{C}$ (isothermal); & flow rate: $1.3 \mathrm{~mL} / \mathrm{min}$ & Arnica alpina \\
\hline
\end{tabular}

Abbreviations: Py, pyridine, BSTFA, N,O-bis(trimethylsilyl)trifluoroacetamide; TMS, trimethylsily; HMDS, hexamethyldisilazane; TMCS, trimethylchlorosilane; APCI-TOF-MS, atmospheric pressure chemical ionization combined with quadrupole - time of flight mass spectrometry; FID, flame ionization detector; QMS, quadrupole mass spectrometry.

Table 7. Counter-current chromatography, as a preparative technique in the separation of luteolin derivatives.

\begin{tabular}{|c|c|c|c|c|c|}
\hline Luteolin Derivative & Solvent System & Conditions & Detection & Analyzed Species & Ref. \\
\hline Luteolin 6-glucoside & $\begin{array}{l}\text { EtOAc: } \mathrm{BuOH}: \mathrm{H}_{2} \mathrm{O} \\
(2: 1: 3 v / v / v)\end{array}$ & $\begin{array}{l}\text { rotation speed: } 800 \mathrm{rpm} ; \\
\text { flow rate (lower phase): } 2.4 \mathrm{~mL} / \mathrm{min}\end{array}$ & $\begin{array}{l}\text { UV-VIS, } 254 \mathrm{~nm} ; \\
\text { NMR; MS }\end{array}$ & Patrinia villosa & {$[173]$} \\
\hline Luteolin 7-glucoside & $\begin{array}{c}\text { EtOAc: } \mathrm{EtOH}: \mathrm{AcOH}: \mathrm{H}_{2} \mathrm{O} \\
(4: 1: 0.25: 5 v / v / v / v)\end{array}$ & $\begin{array}{l}\text { rotation speed: } 800 \mathrm{rpm} ; \\
\text { flow rate (lower phase): } 1.5 \mathrm{~mL} / \mathrm{min}\end{array}$ & $\begin{array}{l}\text { UV, } 254 \mathrm{~nm} ; \\
\text { NMR; MS }\end{array}$ & Paeonia suffruticosa & [177] \\
\hline $\begin{array}{l}\text { Luteolin 6,8-dihexoside } \\
\text { Luteolin 8-glucoside } \\
\text { Luteolin 6-glucoside } \\
\text { Luteolin 7-glucoside }\end{array}$ & $\begin{array}{l}\mathrm{Hx}: \mathrm{EtOH}: \mathrm{H}_{2} \mathrm{O} \\
\quad(4: 3: 1 v / v / v)\end{array}$ & $\begin{array}{l}\text { rotation speed: } 850 \mathrm{rpm} ; \\
\text { flow rate (lower phase): } 2 \mathrm{~mL} / \mathrm{min}\end{array}$ & TLC; HPLC-UV-HRMS & Lippia origanoides & {$[178]$} \\
\hline
\end{tabular}

Abbreviations: $\mathrm{AcOH}$, acetic acid; $\mathrm{BuOH}$, butanol; EtOAc, ethyl acetate; EtOH, ethanol; HRMS, high resolution mass spectrometry; Hx, hexane, rpm, revolutions per minute. 


\section{Conclusions}

The presented comparison of chromatographic methods currently used to determine luteolin and its derivatives provides a systematic summary of the available knowledge. Without a doubt, chromatographic analysis may be successfully employed as an efficient method for both qualitative assessment (fingerprinting) and quantitative determination of luteolin derivatives. In tedious and time-consuming determinations of multi-ingredient plant extracts, including those that contain the most prevalent luteolin derivatives, combinations of large-scale chromatographic techniques with serially aligned detection modalities such as LC-MS/MS or LC/NMR/MS were found to be particularly useful. Such beneficial coupling of chromatography with other analytic techniques expands analytical capabilities while additionally improving the accuracy, sensitivity, and precision of assays. Despite the dominant position of LC in the analysis of natural compounds and the dynamic development of novel chromatographic methods, the TLC/HPTLC has not lost its important place in the phytochemical analysis of luteolin derivatives. The technique is relatively simple and inexpensive while facilitating rapid qualitative and quantitative analysis of test compounds. In addition, it facilitates large quantities of diluted samples being deposited in the stationary phase, allowing for a wider choice of mobile phase carriers. The technique is subject to continuous improvements and its range of applicability is expanding.

Effective chromatographic analysis in the determination of luteolin derivatives requires appropriately selected chromatographic separation conditions. The appropriate choice of stationary phase sorbent may significantly improve test conditions. Most TLC analyses of luteolin derivatives are carried out in normal phase systems featuring a polar (hydrophilic) sorbent phase. In HPLC, separation conditions can be chosen more arbitrarily as the technique allows for the use of stationary phases of varying polarity which is particularly advantageous in the analysis of compounds that are either insoluble or poorly soluble in water. In order to additionally improve the sensitivity of HPLC analyses, one should focus on the column parameters responsible for appropriate separation. Quite often, the fairly successful analyses are used as starting points for further modifications, including the development of preparative-scale analyses.

Of all chromatographic techniques, GC is the least applicable in the analysis of plant extracts, including those that contain luteolin derivatives. Despite its high sensitivity and efficiency when coupled with various detection techniques (MS, MS/MS, TOF-MS), the chromatographic separation process involves high temperatures and derivatization of analytes. Due to this important aspect, GC-MS is less frequently used in the analysis of polyphenolic compounds.

The complexity of plant matrices is unquestionably a significant problem in the chromatographic analysis of plant extracts. It has a negative impact on the efficacy of analyses and prevents complete identification of all components of the plant extract. However, chromatography remains the primary and most effective analytical technique available in the current state of the art for the determination of compounds of natural origin.

Author Contributions: Conceptualization, M.T. and M.Z.-K.; literature investigation, A.M.J.; writing-original draft preparation, A.M.J., M.T., M.Z.-K.; writing-review and editing, A.M.J., M.T.; supervision, M.T., M.Z.-K.

Funding: The work was funded by the project № POWR.03.02.00-00-I051/16 from European Union funds, PO WER 2014-2020, grant №. 05/IMSD/G/2019.

Conflicts of Interest: All authors state that they are free of any conflicts of interest related to this paper.

\section{References}

1. López-Lázaro, M. Distribution and biological activities of the flavonoid luteolin. Mini Rev. Med. Chem. 2009, 9, 31-59. [CrossRef] [PubMed]

2. Szultka, M.; Buszewski, B.; Papaj, K.; Szeja, W.; Rusin, A. Determination of flavonoids and their metabolites by chromatographic techniques. Trends Anal. Chem. 2013, 47, 47-67. [CrossRef] 
3. Nabavi, S.F.; Braidy, N.; Gortzi, O.; Sobarzo-Sanchez, E.; Daglia, M.; Skalicka-Woźniak, K.; Nabavi, S.M. Luteolin as an anti-inflammatory and neuroprotective agent: A brief review. Brain Res. Bull. 2015, 119, 1-11. [CrossRef] [PubMed]

4. Bravo, L. Polyphenols: Chemistry, dietary sources, metabolism, and nutritional significance. Nutr. Rev. 1998, 56, 317-333. [CrossRef]

5. Lin, Y.; Shi, R.; Wang, X.; Shen, H.-M. Luteolin, a flavonoid with potential for cancer prevention and therapy. Curr. Cancer Drug Targets 2008, 8, 634-646. [CrossRef]

6. Aziz, N.; Kim, M.-Y.; Cho, J.Y. Anti-inflammatory effects of luteolin: A review of in vitro, in vivo, and in silico studies. J. Ethnopharmacol. 2018, 225, 342-358. [CrossRef]

7. Imran, M.; Rauf, A.; Abu-Izneid, T.; Nadeem, M.; Shariati, M.A.; Khan, I.A.; Imran, A.; Orhan, I.E.; Rizwan, M.; Atif, M.; et al. Luteolin, a flavonoid, as an anticancer agent: A review. Biomed. Pharmacother. 2019, 112, 108612. [CrossRef]

8. Narayana, K.R.; Reddy, M.S.; Chaluvadi, M.R.; Krishna, D.R. Bioflavonoids classification, pharmacological, biochemical effects and therapeutic potential. Indian J. Pharmacol. 2001, 33, 2-16.

9. Harborne, J.B.; Baxter, H. The Handbook of Natural Flavonoids; Harborne, J.B., Baxter, H., Eds.; John Wiley \& Sons, Inc.: Chichester, UK, 1999.

10. Lam, K.Y.; Ling, A.P.K.; Koh, R.Y.; Wong, Y.P.; Say, Y.H. A review on medicinal properties of orientin. Adv. Pharmacol. Sci. 2016, 2016, 4104595. [CrossRef]

11. Zheng, H.; Zhang, M.; Luo, H.; Li, H. Isoorientin alleviates UVB-induced skin injury by regulating mitochondrial ROS and cellular autophagy. Biochem. Biophys. Res. Commun. 2019, 514, 1133-1139. [CrossRef]

12. Wu, Q.-Y.; Wong, Z.C.-F.; Wang, C.; Fung, A.H.-Y.; Wong, E.O.-Y.; Chan, G.K.-L.; Dong, T.T.-X.; Chen, Y.; Tsim, K.W.-K. Isoorientin derived from Gentiana veitchiorum Hemsl. flowers inhibit melanogenesis by down-regulating MITF-induced tyrosinase expression. Phytomedicine 2019, 57, 129-136. [CrossRef] [PubMed]

13. Fan, X.; Lv, H.; Wang, L.; Deng, X.; Ci, X. Isoorientin ameliorates APAP-induced hepatotoxicity via activation Nrf2 antioxidative pathway: The involvement of AMPK/Akt/GSK3ß. Front. Pharmacol. 2018, 9, 1334. [CrossRef] [PubMed]

14. Anilkumar, K.; Reddy, G.V.; Azad, R.; Yarla, N.S.; Dharmapuri, G.; Srivastava, A.; Kamal, M.A.; Pallu, R. Evaluation of anti-inflammatory properties of isoorientin isolated from tubers of Pueraria tuberosa. Oxid. Med. Cell. Longev. 2017, 2017, 5498054. [CrossRef]

15. Jo, B.-G.; Park, N.-J.; Jegal, J.; Choi, S.; Lee, S.W.; Yi, L.W.; Kim, S.-N.; Yang, M.H. Stellera chamaejasme and its main compound luteolin 7-O-glucoside alleviates skin lesions in oxazolone- and 2,4-dinitrochlorobenzene-stimulated murine models of atopic dermatitis. Planta Med. 2018, 85, 583-590. [CrossRef] [PubMed]

16. Chen, S.; Yang, B.; Xu, Y.; Rong, Y.; Qiu, Y. Protection of luteolin-7-O-glucoside against apoptosis induced by hypoxia/reoxygenation through the MAPK pathways in H9c2 cells. Mol. Med. Rep. 2018, 17, 7156-7162. [CrossRef]

17. McNair, H.M.; Miller, J.M. Basic Gas Chromatography, 2nd ed.; John Wiley \& Sons, Inc.: Hoboken, NJ, USA, 2009.

18. Monteiro, M.L.G.; Marsico, E.; Lázaro, C.; Conte Júnior, C. Thin-layer chromatography applied to foods of animal origin: A tutorial review. J. Anal. Chem. 2016, 71, 459-470. [CrossRef]

19. Kazakevich, Y.; LoBrutto, R. HPLC theory and practice. In HPLC for Pharmaceutical Scientists; Kazakevich, Y., LoBrutto, R., Eds.; John Wiley \& Sons, Inc.: Hoboken, NJ, USA, 2007; pp. 3-13.

20. Medić-Šarić, M.; Jasprica, I.; Mornar, A.; Maleš, Ž. Application of TLC in the isolation and analysis of flavonoids. In Thin Layer Chromatography in Phytochemistry; Waksmundzka-Hajnos, M., Shrema, J., Kowalska, T., Eds.; CRC Press, Taylor \& Francis Group: Boca Raton, FL, USA, 2008; pp. 405-423.

21. Fuchs, B.; Sü $\beta$, R.; Teuber, K.; Eibisch, M.; Schiller, J. Lipid analysis by thin-layer chromatography-A review of the current state. J. Chromatogr. A 2011, 1218, 2754-2774. [CrossRef]

22. Poole, C.F. Milestones, core concepts, and contrasts. In Instrumental Thin-Layer Chromatography; Poole, C.F., Ed.; Elsevier Inc.: Amsterdam, The Netherlands, 2015; pp. 1-3.

23. Santiago, M.; Strobel, S. Thin layer chromatography. Methods Enzymol. 2013, 533, 303-324.

24. Waksmundzka-Hajnos, M.; Hawrył, M.A.; Cieśla, Ł. Analysis of plant material. In Instrumental Thin-Layer Chromatography; Poole, C.F., Ed.; Elsevier Inc.: Amsterdam, The Netherlands, 2015; pp. 505-508. 
25. Phadungrakwittaya, R. Identification of apigenin and luteolin in Artemisia annua L. for the quality control. Siriraj Med. J. 2019, 71, 240-245. [CrossRef]

26. Mučaji, P.; Nagy, M.; Liptaj, T.; Prónayová, N.; Švajdlenka, E. Separation of a mixture of luteolin-7-rutinoside and luteolin-7-neohesperidoside isolated from Ligustrum vulgare L. JPC J. Planar Chromatogr. 2009, 22, 301-304. [CrossRef]

27. Aghakhani, F.; Kharazian, N.; Lori Gooini, Z. Flavonoid constituents of Phlomis (Lamiaceae) species using liquid chromatography mass spectrometry. Phytochem. Anal. 2018, 29, 180-195. [CrossRef] [PubMed]

28. Kozyra, M.; Głowniak, K.; Boguszewska, M. The analysis of flavonoids in the flowering herbs of Carduus acanthoides L. Curr. Issues Pharm. Med. Sci. 2013, 26, 10-15.

29. Schwaiger, S.; Seger, C.; Wiesbauer, B.; Schneider, P.; Ellmerer, E.P.; Sturm, S.; Stuppner, H. Development of an HPLC-PAD-MS assay for the identification and quantification of major phenolic edelweiss (Leontopodium alpium Cass.) constituents. Phytochem. Anal. 2006, 17, 291-298. [CrossRef] [PubMed]

30. Jesionek, W.; Majer-Dziedzic, B.; Choma, I. Separation, identification, and investigation of antioxidant ability of plant extract components using TLC, LC-MS, and TLC-DPPH. J. Liq. Chromatogr. Relat. Technol. 2015, 38, 1147-1153. [CrossRef]

31. Amirkhanova, A.; Ustenova, G.; Krauze, M.; Poblocka, L.; Shynykul, Z. Thin-layer chromatography analysis of extract Oxytropis glabra lam. dc. In Proceedings of the 18th International Multidisciplinary Scientific Geoconference (SGEM 2018), Albena, Bulgaria, 30 June-9 July 2018; pp. 775-782.

32. Nile, S.; Park, S.W. HPTLC densitometry method for simultaneous determination of flavonoids in selected medicinal plants. Front. Life Sci. 2015, 8, 97-103. [CrossRef]

33. Kukkar, M.; Kukkar, R.; Saluja, A. Validation of HPTLC method for the analysis of luteolin in Cardiospermum halicacabum Linn. Int. J. Green Pharm. 2014, 8, 252-256. [CrossRef]

34. Hassanein, H.; Said-Al Ahl, H.; MM, A. Antioxidant polyphenolic constituents of Satureja montana L. Growing in Egypt. Int. J. Pharm. Pharm. Sci. 2014, 6, 578-581.

35. Satpathy, S.; Patra, A.; Ahirwar, B. Development and validation of a novel high-performance thin-layer chromatography method for the simultaneous determination of apigenin and luteolin in Hygrophila spinosa T. Anders. JPC J. Planar Chromatogr. Mod. TLC 2018, 31, 437-443. [CrossRef]

36. Shawky, E.; Abou El Kheir, R.M. Rapid discrimination of different Apiaceae species based on HPTLC fingerprints and targeted flavonoids determination using multivariate image analysis. Phytochem. Anal. 2018, 29, 452-462. [CrossRef]

37. Swar, G.; Shailajan, S.; Menon, S. Activity based evaluation of a traditional Ayurvedic medicinal plant: Saraca asoca (Roxb.) de Wilde flowers as estrogenic agents using ovariectomized rat model. J. Ethnopharmacol. 2017, 195, 324-333. [CrossRef]

38. Patel, N.; Patel, K.; Patel, K.; Tejal, G. Validated HPTLC method for quantification of luteolin and apigenin in Premna mucronata Roxb., Verbenaceae. Adv. Pharmacol. Sci. 2015, 2015, 1-7. [CrossRef] [PubMed]

39. Gupta, N.; Lobo, R.; Kumar, N.; Bhagat, J.K.; Mathew, J.E. Identity-based High-performance thin layer chromatography fingerprinting profile and tumor inhibitory potential of Anisochilus carnosus (L.f.) wall against ehrlich ascites carcinoma. Pharmacogn. Mag. 2015, 11, 474-480.

40. Bros, I.; Soran, M.-L.; Naşcu-Briciu, R.; Codruta, C. HPTLC quantification of some flavonoids in extracts of Satureja hortensis L. obtained by use of different techniques. JPC J. Planar Chromatogr. 2009, 22, 25-28. [CrossRef]

41. Ristivojevic, P.; Dimkic, I.; Trifkovic, J.; Beric, T.; Vovk, I.; Milojkovic-Opsenica, D.; Stankovic, S. Antimicrobial activity of Serbian propolis evaluated by means of MIC, HPTLC, bioautography and chemometrics. PLoS ONE 2016, 11, e0157097. [CrossRef] [PubMed]

42. Shaikh, F.; Sancheti, J.; Sathaye, S. Phytochemical and pharmacological investigations of Eclipta alba (Linn.) Hassak leaves for antiepileptic activity. Int. J. Pharm. Pharm. Sci. 2012, 4, 319-323.

43. Margină, D.; Olaru, O.T.; Ilie, M.; Gradinaru, D.; GuTu, C.; Voicu, S.; Dinischiotu, A.; Spandidos, D.A.; Tsatsakis, A.M. Assessment of the potential health benefits of certain total extracts from Vitis vinifera, Aesculus hyppocastanum and Curcuma longa. Exp. Ther. Med. 2015, 10, 1681-1688. [CrossRef]

44. Chelyn, J.L.; Omar, M.H.; Mohd Yousof, N.S.A.; Ranggasamy, R.; Wasiman, M.I.; Ismail, Z. Analysis of flavone C-glycosides in the leaves of Clinacanthus nutans (Burm. f.) Lindau by HPTLC and HPLC-UV/DAD. Sci. World J. 2014, 2014, 1-6. [CrossRef] 
45. Pereira, C.A.M.; Yariwake, J.H.; Lancas, F.M.; Wauters, J.-N.; Tits, M.; Angenot, L. A HPTLC densitometric determination of flavonoids from Passiflora alata, P. edulis, P. incarnata and P. caerulea and comparison with HPLC method. Phytochem. Anal. 2004, 15, 241-248. [CrossRef]

46. Wang, J.; Tang, F.; Yue, Y.; Guo, X.; Yao, X. Development and validation of an HPTLC method for simultaneous quantitation of isoorientin, isovitexin, orientin, and vitexin in bamboo-leaf flavonoids. J. AOAC Int. 2010, 93, 1376-1383.

47. Cook, R.; Hennell, J.R.; Lee, S.; Khoo, C.S.; Carles, M.C.; Higgins, V.J.; Govindaraghavan, S.; Sucher, N.J. The Saccharomyces cerevisiae transcriptome as a mirror of phytochemical variation in complex extracts of Equisetum arvense from America, China, Europe and India. BMC Genomics 2013, 14, 445. [CrossRef]

48. Srinivas, P.; Reddy, S.R. Screening for antibacterial principle and activity of Aerva javanica (Burm.f) Juss. ex Schult. Asian Pac. J. Trop. Biomed. 2012, 2, S838-S845. [CrossRef]

49. Guerrini, A.; Bruni, R.; Maietti, S.; Poli, F.; Rossi, D.; Paganetto, G.; Muzzoli, M.; Scalvenzi, L.; Sacchetti, G. Ecuadorian stingless bee (Meliponinae) honey: A chemical and functional profile of an ancient health product. Food Chem. 2009, 114, 1413-1420. [CrossRef]

50. Bazylko, A.; Tomczyk, M.; Flazińska, A.; Lȩgas, A. Chemical fingerprint of Potentilla species by using HPTLC method. JPC J. Planar Chromatogr. 2011, 24, 441-444. [CrossRef]

51. Celep, E.; Akyüz, S.; İnan, Y.; Yesilada, E. Assessment of potential bioavailability of major phenolic compounds in Lavandula stoechas L. ssp. stoechas. Ind. Crops Prod. 2018, 118, 111-117. [CrossRef]

52. Bilušić Vundać, V.; Maleš, Ž.; Plazibat, M.; Golja, P.; Cetina-Čižmek, B. HPTLC determination of flavonoids and phenolic acids in some Croatian Stachys taxa. JPC J. Planar Chromatogr. 2005, 18, 269-273. [CrossRef]

53. Fecka, I.; Kowalczyk, A.; Cisowski, W. Optimization of the separation of flavonoid glycosides and rosmarinic acid from Mentha piperita on HPTLC plates. JPC J. Planar Chromatogr. 2004, 17, 22-25. [CrossRef]

54. Fecka, I.; Turek, S. Determination of water-soluble polyphenolic compounds in commercial herbal teas from Lamiaceae: Peppermint, melissa, and sage. J. Agric. Food Chem. 2007, 55, 10908-10917. [CrossRef]

55. Fecka, I.; Turek, S. Determination of polyphenolic compounds in commercial herbal drugs and spices from Lamiaceae: Thyme, wild thyme and sweet marjoram by chromatographic techniques. Food Chem. 2008, 108, 1039-1053. [CrossRef]

56. Fecka, I.; Raj, D.; Krauze-Baranowska, M. Quantitative determination of four water-soluble compounds in herbal drugs from Lamiaceae using different chromatographic techniques. Chromatographia 2007, 66, 87-93. [CrossRef]

57. Jug, U.; Glavnik, V.; Kranjc, E.; Vovk, I. HPTLC-densitometric and HPTLC-MS methods for analysis of flavonoids. J. Liq. Chromatogr. Relat. Technol. 2018, 41, 329-341. [CrossRef]

58. Lebot, V.; Lawac, F.; Michalet, S.; Legendre, L. Characterization of taro [Colocasia esculenta (L.) Schott] germplasm for improved flavonoid composition and content. Plant. Genet. Resour. 2017, 15, 260-268. [CrossRef]

59. Orsini, F.; Vovk, I.; Glavnik, V.; Jug, U.; Corradini, D. HPTLC, HPTLC-MS/MS and HPTLC-DPPH methods for analyses of flavonoids and their antioxidant activity in Cyclanthera pedata leaves, fruits and dietary supplement. J. Liq. Chromatogr. Relat. Technol. 2019, 42, 290-301. [CrossRef]

60. Nile, S.H.; Park, S.W. HPTLC analysis, antioxidant and antigout activity of Indian plants. Iran. J. Pharm. Res. 2014, 13, 531-539. [PubMed]

61. Chisvert, A.; Benedé, J.L.; Salvador, A. Current trends on the determination of organic UV filters in environmental water samples based on microextraction techniques-A review. Anal. Chim. Acta 2018, 1034, 22-38. [CrossRef]

62. Dias, I.H.K.; Wilson, S.R.; Roberg-Larsen, H. Chromatography of oxysterols. Biochimie 2018, 153, 3-12. [CrossRef]

63. Pascual-Maté, A.; Osés, S.M.; Fernández-Muiño, M.A.; Sancho, M.T. Analysis of polyphenols in honey: Extraction, separation and quantification procedures. Sep. Purif. Rev. 2018, 47, 142-158. [CrossRef]

64. Kazakevich, Y. HPLC theory. In HPLC for Pharmaceutical Scientists; Kazakevich, Y., LoBrutto, R., Eds.; John Wiley \& Sons, Inc.: Hoboken, NJ, USA, 2007; pp. 40-70.

65. Kazakevich, Y.; LoBrutto, R. Stationary phases. In HPLC for Pharmaceutical Scientists; Kazakevich, Y., LoBrutto, R., Eds.; John Wiley \& Sons, Inc.: Hoboken, NJ, USA, 2007; pp. 77-90. 
66. Pereira, E.; Barros, L.; Calhelha, R.C.; Dueñas, M.; Carvalho, A.M.; Santos-Buelga, C.; Ferreira, I.C.F.R. Bioactivity and phytochemical characterization of Arenaria montana L. Food Funct. 2014, 5, 1848-1855. [CrossRef]

67. Barros, L.; Dueñas, M.; Carvalho, A.M.; Ferreira, I.C.F.R.; Santos-Buelga, C. Characterization of phenolic compounds in flowers of wild medicinal plants from Northeastern Portugal. Food Chem. Toxicol. 2012, 50, 1576-1582. [CrossRef]

68. Dias, M.I.; Barros, L.; Dueñas, M.; Pereira, E.; Carvalho, A.M.; Alves, R.C.; Oliveira, M.B.P.P.; Santos-Buelga, C.; Ferreira, I.C.F.R. Chemical composition of wild and commercial Achillea millefolium L. and bioactivity of the methanolic extract, infusion and decoction. Food Chem. 2013, 141, 4152-4160. [CrossRef]

69. Rodrigues, S.; Calhelha, R.C.; Barreira, J.C.M.; Dueñas, M.; Carvalho, A.M.; Abreu, R.M.V.; Santos-Buelga, C.; Ferreira, I.C.F.R. Crataegus monogyna buds and fruits phenolic extracts: Growth inhibitory activity on human tumor cell lines and chemical characterization by HPLC-DAD-ESI/MS. Food Res. Int. 2012, 49, 516-523. [CrossRef]

70. Roriz, C.L.; Barros, L.; Carvalho, A.M.; Santos-Buelga, C.; Ferreira, I.C.F.R. Pterospartum tridentatum, Gomphrena globosa and Cymbopogon citratus: A phytochemical study focused on antioxidant compounds. Food Res. Int. 2014, 62, 684-693. [CrossRef]

71. Barros, L.; Pereira, E.; Calhelha, R.C.; Dueñas, M.; Carvalho, A.M.; Santos-Buelga, C.; Ferreira, I.C.F.R. Bioactivity and chemical characterization in hydrophilic and lipophilic compounds of Chenopodium ambrosioides L. J. Funct. Foods 2013, 5, 1732-1740. [CrossRef]

72. Viacava, G.E.; Roura, S.I.; López-Márquez, D.M.; Berrueta, L.A.; Gallo, B.; Alonso-Salces, R.M. Polyphenolic profile of butterhead lettuce cultivar by ultrahigh performance liquid chromatography coupled online to UV-visible spectrophotometry and quadrupole time-of-flight mass spectrometry. Food Chem. 2018, 260, 239-273. [CrossRef] [PubMed]

73. Yang, X.; Cui, X.; Zhao, L.; Guo, D.; Feng, L.; Wei, S.; Zhao, C.; Huang, D. Exogenous glycine nitrogen enhances accumulation of glycosylated flavonoids and antioxidant activity in lettuce (Lactuca sativa L.). Front. Plant. Sci. 2017, 8, 2098. [CrossRef]

74. Farag, M.A.; Handoussa, H.; Fekry, M.I.; Wessjohann, L.A. Metabolite profiling in 18 Saudi date palm fruit cultivars and their antioxidant potential via UPLC-qTOF-MS and multivariate data analyses. Food Funct. 2016, 7, 1077-1086. [CrossRef]

75. Ibrahim, R.M.; El-Halawany, A.M.; Saleh, D.O.; El Naggar, E.M.B.; El-Shabrawy, A.E.-R.O.; El-Hawary, S.S. HPLC-DAD-MS/MS profiling of phenolics from Securigera securidaca flowers and its anti-hyperglycemic and anti-hyperlipidemic activities. Rev. Bras. Farmacogn. 2015, 25, 134-141. [CrossRef]

76. Mudrić, S.Ž.; Gašić, U.M.; Dramićanin, A.M.; Ćirić, I.Ž.; Milojković-Opsenica, D.M.; Popović-Đorđević, J.B.; Momirović, N.M.; Tešić, Ž.L. The polyphenolics and carbohydrates as indicators of botanical and geographical origin of Serbian autochthonous clones of red spice paprika. Food Chem. 2017, 217, 705-715. [CrossRef]

77. Barros, L.; Dueñas, M.; Dias, M.I.; Sousa, M.J.; Santos-Buelga, C.; Ferreira, I.C.F.R. Phenolic profiles of in vivo and in vitro grown Coriandrum sativum L. Food Chem. 2012, 132, 841-848. [CrossRef]

78. Quirantes-Pine, R.; Lozano-Sanchez, J.; Herrero, M.; Ibanez, E.; Segura-Carretero, A.; Fernandez-Gutierrez, A. HPLC-ESI-QTOF-MS as a powerful analytical tool for characterising phenolic compounds in olive-leaf extracts. Phytochem. Anal. 2013, 24, 213-223. [CrossRef]

79. Costa, G.; Nunes, F.; Vitorino, C.; Sousa, J.J.; Figueiredo, I.V.; Batista, M.T. Validation of a RP-HPLC method for quantitation of phenolic compounds in three different extracts from Cymbopogon citratus. Res. J. Med. Plant. 2015, 9, 331-339.

80. Liu, G.; Linwu, Z.; Song, D.; Lu, C.; Xu, X. Isolation, purification, and identification of the main phenolic compounds from leaves of celery (Apium graveolens L. var. dulce Mill./Pers.). J. Sep. Sci. 2016, 40, 472-479. [CrossRef] [PubMed]

81. Taghouti, M.; Martins-Gomes, C.; Schäfer, J.; Félix, L.M.; Santos, J.A.; Bunzel, M.; Nunes, F.M.; Silva, A.M. Thymus pulegioides L. as a rich source of antioxidant, anti-proliferative and neuroprotective phenolic compounds. Food Funct. 2018, 9, 3617-3629. [CrossRef] [PubMed]

82. Achour, M.; Mateos, R.; Ben Fredj, M.; Mtiraoui, A.; Bravo, L.; Saguem, S. A comprehensive characterisation of rosemary tea obtained from Rosmarinus officinalis L. collected in a sub-humid area of Tunisia. Phytochem. Anal. 2018, 29, 87-100. [CrossRef] [PubMed] 
83. Ferreres, F.; Gomes, N.G.M.; Valentão, P.; Pereira, D.M.; Gil-Izquierdo, A.; Araújo, L.; Silva, T.C.; Andrade, P.B. Leaves and stem bark from Allophylus africanus P. Beauv.: An approach to anti-inflammatory properties and characterization of their flavonoid profile. Food Chem. Toxicol. 2018, 118, 430-438. [CrossRef]

84. Ravisankar, S.; Abegaz, K.; Awika, J.M. Structural profile of soluble and bound phenolic compounds in teff (Eragrostis tef) reveals abundance of distinctly different flavones in white and brown varieties. Food Chem. 2018, 263, 265-274. [CrossRef]

85. Khallouki, F.; Breuer, A.; Merieme, E.; Ulrich, C.M.; Owen, R.W. Characterization and quantitation of the polyphenolic compounds detected in methanol extracts of Pistacia atlantica Desf. fruits from the Guelmim region of Morocco. J. Pharm. Biomed. Anal. 2017, 134, 310-318. [CrossRef]

86. Vasić, V.; Gašić, U.; Stanković, D.; Lušić, D.; Vukić-Lušić, D.; Milojković-Opsenica, D.; Tešić, Ž.; Trifković, J. Towards better quality criteria of European honeydew honey: Phenolic profile and antioxidant capacity. Food Chem. 2019, 274, 629-641. [CrossRef]

87. Ammar, S.; Contreras, M.d.M.; Belguith-Hadrich, O.; Bouaziz, M.; Segura-Carretero, A. New insights into the qualitative phenolic profile of Ficus carica L. fruits and leaves from Tunisia using ultra-high-performance liquid chromatography coupled to quadrupole-time-of-flight mass spectrometry and their antioxidant activity. RSC Adv. 2015, 5, 20035-20050. [CrossRef]

88. Obmann, A.; Purevsuren, S.; Zehl, M.; Kletter, C.; Reznicek, G.; Narantuya, S.; Glasl, S. HPLC determination of flavonoid glycosides in Mongolian Dianthus versicolor Fisch. (Caryophyllaceae) compared with quantification by UV spectrophotometry. Phytochem. Anal. 2012, 23, 254-259. [CrossRef]

89. Chen, H.-J.; Inbaraj, B.S.; Chen, B.-H. Determination of phenolic acids and flavonoids in Taraxacum formosanum Kitam by liquid chromatography-tandem mass spectrometry coupled with a post-column derivatization technique. Int. J. Mol. Sci. 2012, 13, 260-285. [CrossRef]

90. Tebboub, O.; Cotugno, R.; Oke-Altuntas, F.; Bouheroum, M.; Demirtas, Í.; D'Ambola, M.; Malafronte, N.; Vassallo, A. Antioxidant potential of herbal preparations and components from Galactites elegans (All.) Nyman ex soldano. Evid. Based. Complement. Alternat. Med. 2018, 2018, 9294358. [CrossRef] [PubMed]

91. Saleem, H.; Htar, T.T.; Naidu, R.; Nawawi, N.S.; Ahmad, I.; Ashraf, M.; Ahemad, N. Biological, chemical and toxicological perspectives on aerial and roots of Filago germanica (L.) huds: Functional approaches for novel phyto-pharmaceuticals. Food Chem. Toxicol. 2019, 123, 363-373. [CrossRef] [PubMed]

92. Tahri, W.; Chatti, A.; Romero-González, R.; López-Gutiérrez, N.; Frenich, A.G.; Landoulsi, A. Phenolic profiling of the aerial part of Chrysanthemum trifurcatum using ultra high-performance liquid chromatography coupled to Orbitrap high resolution mass spectrometry. Anal. Methods 2016, 8, 3517-3527. [CrossRef]

93. Dall'Acqua, S.; Peron, G.; Ferrari, S.; Gandin, V.; Bramucci, M.; Quassinti, L.; Mártonfi, P.; Maggi, F. Phytochemical investigations and antiproliferative secondary metabolites from Thymus alternans growing in Slovakia. Pharm. Biol. 2017, 55, 1162-1170. [CrossRef]

94. Abu-Reidah, I.M.; Gil-Izquierdo, Á.; Medina, S.; Ferreres, F. Phenolic composition profiling of different edible parts and by-products of date palm (Phoenix dactylifera L.) by using HPLC-DAD-ESI/MSn. Food Res. Int. 2017, 100, 494-500. [CrossRef]

95. Vinayagam, A.; Sudha, P.N. Separation and identification of phenolic acid and flavonoids from Nerium indicum flowers. Indian J. Pharm. Sci. 2015, 77, 91-95. [CrossRef]

96. Vitalini, S.; Madeo, M.; Tava, A.; Iriti, M.; Vallone, L.; Avato, P.; Cocuzza, C.E.; Simonetti, P.; Argentieri, M.P. Chemical profile, antioxidant and antibacterial activities of Achillea moschata Wulfen, an endemic species from the Alps. Molecules 2016, 21, 830. [CrossRef]

97. Liu, H.; Zhang, M.; Guo, Y.; Qiu, H. Solid-phase extraction of flavonoids in honey samples using carbamate-embedded triacontyl-modified silica sorbent. Food Chem. 2016, 204, 56-61. [CrossRef]

98. Li, L.; Jiang, H.; Wu, H.; Zeng, S. Simultaneous determination of luteolin and apigenin in dog plasma by RP-HPLC. J. Pharm. Biomed. Anal. 2005, 37, 615-620. [CrossRef]

99. Klangprapun, S.; Buranrat, B.; Caichompoo, W.; Nualkaew, S. Pharmacognostical and physicochemical studies of Enhalus acoroides (L.F.) royle (rhizome). Pharmacogn. J. 2018, 10, s89-s94. [CrossRef]

100. Song, T.; Liu, L. A strategy for quality control of the fruits of Perilla frutescens (L.) Britt based on antioxidant activity and fingerprint analysis. Anal. Methods 2016, 8, 295-302. [CrossRef]

101. da Silva, J.B.; dos Temponi, V.S.; Gasparetto, C.M.; Fabri, R.L.; de Aragão, D.M.O.; de Pinto, N.C.C.; Ribeiro, A.; Scio, E.; Del-Vechio-Vieira, G.; de Sousa, O.V.; et al. Vernonia condensata Baker (Asteraceae): A promising source of antioxidants. Oxid. Med. Cell. Longev. 2013, 2013, 698018. [CrossRef] [PubMed] 
102. Hasan, H.T.; Kadhim, E.J. Phytochemical investigation of leaves and seeds of Corchorus olitorius L. Cultivated in Iraq. Asian J. Pharm. Clin. Res. 2018, 11, 408-417. [CrossRef]

103. Yin, L.; Han, H.; Zheng, X.; Wang, G.; Li, Y.; Wang, W. Flavonoids analysis and antioxidant, antimicrobial, and anti-inflammatory activities of crude and purified extracts from Veronicastrum latifolium. Ind. Crops Prod. 2019, 137, 652-661. [CrossRef]

104. Amanpour, A.; Kelebek, H.; Selli, S. LC-DAD-ESI-MS/MS-based phenolic profiling and antioxidant activity in Turkish cv. Nizip Yaglik olive oils from different maturity olives. J. Mass Spectrom. 2019, 54, 227-238. [CrossRef]

105. Ivanov, I.; Vrancheva, R.; Petkova, N.; Tumbarski, Y.; Dincheva, I.; Badjakov, I. Phytochemical compounds of anise hyssop (Agastache foeniculum) and antibacterial, antioxidant, and acetylcholinesterase inhibitory properties of its essential oil. J. Appl. Pharm. Sci. 2019, 9, 72-78.

106. Ren, G.; Xue, P.; Sun, X.; Zhao, G. Determination of the volatile and polyphenol constituents and the antimicrobial, antioxidant, and tyrosinase inhibitory activities of the bioactive compounds from the by-product of Rosa rugosa Thunb. var. plena Regal tea. BMC Complement. Altern. Med. 2018, 18, 307. [CrossRef]

107. Tomczyk, M.; Gudej, J. Quantitative analysis of flavonoids in the flowers and leaves of Ficaria verna Huds. Z. Naturforsch. C. 2003, 58, 762-764. [CrossRef]

108. Félix-Silva, J.; Gomes, J.A.S.; Fernandes, J.M.; Moura, A.K.C.; Menezes, Y.A.S.; Santos, E.C.G.; Tambourgi, D.V.; Silva-Junior, A.A.; Zucolotto, S.M.; Fernandes-Pedrosa, M.F. Comparison of two Jatropha species (Euphorbiaceae) used popularly to treat snakebites in Northeastern Brazil: Chemical profile, inhibitory activity against Bothrops erythromelas venom and antibacterial activity. J. Ethnopharmacol. 2018, 213, 12-20. [CrossRef]

109. Sánchez-Roque, Y.; Ayora-Talavera, G.; Rincón-Rosales, R.; Gutiérrez-Miceli, F.; Meza Gordillo, R.; Winkler, R.; Gamboa-Becerra, R.; Ayora, T.; Ruíz-Valdiviezo, V. The flavonoid fraction from Rhoeo discolor leaves acts antiviral against influenza a virus. Rec. Nat. Prod. 2017, 11, 532-546. [CrossRef]

110. Reheman, A.; Aisa, H.A.; Ma, Q.L.; Nijat, D.; Abdulla, R. Quality evaluation of the traditional medicine majun mupakhi ELA via chromatographic fingerprinting coupled with UHPLC-DAD-Quadrupole-Orbitrap-MS and the antioxidant activity in vitro. Evid. Based. Complement. Alternat. Med. 2018, 2018, 1035809. [CrossRef] [PubMed]

111. Moreno-González, R.; Juan, M.E.; Planas, J.M. Table olive polyphenols: A simultaneous determination by liquid chromatography-mass spectrometry. J. Chromatogr. A 2019, 460434. [CrossRef] [PubMed]

112. Luca, S.V.; Miron, A.; Aprotosoaie, A.C.; Mihai, C.-T.; Vochita, G.; Gherghel, D.; Ciocarlan, N.; Skalicka-Wozniak, K. HPLC-DAD-ESI-Q-TOF-MS/MS profiling of Verbascum ovalifolium Donn ex Sims and evaluation of its antioxidant and cytogenotoxic activities. Phytochem. Anal. 2019, 30, 34-45. [CrossRef] [PubMed]

113. Quirantes-Piné, R.; Funes, L.; Micol, V.; Segura-Carretero, A.; Fernández-Gutiérrez, A. High-performance liquid chromatography with diode array detection coupled to electrospray time-of-flight and ion-trap tandem mass spectrometry to identify phenolic compounds from a Lemon verbena extract. J. Chromatogr. A 2009, 1216, 5391-5397. [CrossRef] [PubMed]

114. Flores-Ocelotl, M.R.; Rosas-Murrieta, N.H.; Moreno, D.A.; Vallejo-Ruiz, V.; Reyes-Leyva, J.; Dominguez, F.; Santos-López, G. Taraxacum officinale and Urtica dioica extracts inhibit dengue virus serotype 2 replication in vitro. BMC Complement. Altern. Med. 2018, 18, 95. [CrossRef] [PubMed]

115. Colombo, R.; Harumi Yariwake, J.; Queiroz, E.; Ndjoko, K.; Hostettmann, K. LC-MS/MS analysis of sugarcane extracts and differentiation of monosaccharides moieties of flavone C-glycosides. J. Liq. Chromatogr. Relat. Technol. 2013, 36, 239-258. [CrossRef]

116. Elkady, W.M.; Ibrahim, E.A.; Gonaid, M.H.; El Baz, F.K. Chemical profile and biological activity of Casimiroa edulis non-edible fruit's parts. Adv. Pharm. Bull. 2017, 7, 655-660. [CrossRef]

117. Mattila, P.; Astola, J.; Kumpulainen, J. Determination of flavonoids in plant material by HPLC with diode-array and electro-array detections. J. Agric. Food Chem. 2000, 48, 5834-5841. [CrossRef]

118. de Beer, D.; Joubert, E.; Malherbe, C.J.; Jacobus Brand, D. Use of countercurrent chromatography during isolation of 6-hydroxyluteolin-7-O- $\beta$-glucoside, a major antioxidant of Athrixia phylicoides. J. Chromatogr. A 2011, 1218, 6179-6186. [CrossRef] 
119. Ziani, B.E.C.; Barros, L.; Boumehira, A.Z.; Bachari, K.; Heleno, S.A.; Alves, M.J.; Ferreira, I.C.F.R. Profiling polyphenol composition by HPLC-DAD-ESI/MSn and the antibacterial activity of infusion preparations obtained from four medicinal plants. Food Funct. 2018, 9, 149-159. [CrossRef]

120. Bessada, S.M.F.; Barreira, J.C.M.; Barros, L.; Ferreira, I.C.F.R.; Oliveira, M.B.P.P. Phenolic profile and antioxidant activity of Coleostephus myconis (L.) Rchb.f.: An underexploited and highly disseminated species. Ind. Crops Prod. 2016, 89, 45-51. [CrossRef]

121. Llorent-Martínez, E.J.; Ortega-Barrales, P.; Zengin, G.; Uysal, S.; Ceylan, R.; Guler, G.O.; Mocan, A.; Aktumsek, A. Lathyrus aureus and Lathyrus pratensis: Characterization of phytochemical profiles by liquid chromatography-mass spectrometry, and evaluation of their enzyme inhibitory and antioxidant activities. RSC Adv. 2016, 6, 88996-89006. [CrossRef]

122. Carvalho, A.R.; Costa, G.; Figueirinha, A.; Liberal, J.; Prior, J.A.V.; Lopes, M.C.; Cruz, M.T.; Batista, M.T. Urtica spp.: Phenolic composition, safety, antioxidant and anti-inflammatory activities. Food Res. Int. 2017, 99, 485-494. [CrossRef] [PubMed]

123. Brito, A.; Ramirez, J.E.; Areche, C.; Sepúlveda, B.; Simirgiotis, M.J. HPLC-UV-MS profiles of phenolic compounds and antioxidant activity of fruits from three citrus species consumed in Northern Chile. Molecules 2014, 19, 17400-17421. [CrossRef]

124. Plazonić, A.; Bucar, F.; Maleš, Ž.; Mornar, A.; Nigović, B.; Kujundžić, N. Identification and quantification of flavonoids and phenolic acids in burr parsley (Caucalis platycarpos L.), using high-performance liquid chromatography with diode array detection and electrospray ionization mass spectrometry. Molecules 2009, 14, 2466-2490. [CrossRef]

125. Pereira, O.R.; Catarino, M.D.; Afonso, A.F.; Silva, A.M.S.; Cardoso, S.M. Salvia elegans, Salvia greggii and Salvia officinalis decoctions: Antioxidant activities and inhibition of carbohydrate and lipid metabolic enzymes. Molecules 2018, 23, 3169. [CrossRef]

126. Afonso, A.F.; Pereira, O.R.; Neto, R.T.; Silva, A.M.S.; Cardoso, S.M. Health-promoting effects of Thymus herba-barona, Thymus pseudolanuginosus, and Thymus caespititius decoctions. Int. J. Mol. Sci. 2017, 18, 1879. [CrossRef]

127. Ferreres, F.; Grosso, C.; Gil-Izquierdo, A.; Fernandes, A.; Valentão, P.; Andrade, P. Comparing the phenolic profile of Pilocarpus pennatifolius Lem. by HPLC-DAD-ESI/MSn with respect to authentication and enzyme inhibition potential. Ind. Crops Prod. 2015, 77, 391-401. [CrossRef]

128. Otify, A.; George, C.; Elsayed, A.; Farag, M.A. Mechanistic evidence of Passiflora edulis (Passifloraceae) anxiolytic activity in relation to its metabolite fingerprint as revealed via LC-MS and chemometrics. Food Funct. 2015, 6, 3807-3817. [CrossRef]

129. Afifi, F.U.; Kasabri, V.; Litescu, S.; Abaza, I.F.; Tawaha, K. Phytochemical and biological evaluations of Arum hygrophilum Boiss. (Araceae). Pharmacogn. Mag. 2017, 13, 275-280. [CrossRef]

130. Kowalska, I.; Pecio, L.; Ciesla, L.; Oleszek, W.; Stochmal, A. Isolation, chemical characterization, and free radical scavenging activity of phenolics from Triticum aestivum L. aerial parts. J. Agric. Food Chem. 2014, 62, 11200-11208. [CrossRef]

131. Kostic, A.Z.; Gasic, U.M.; Pesic, M.B.; Stanojevic, S.P.; Barac, M.B.; Macukanovic-Jocic, M.P.; Avramov, S.N.; Tesic, Z.L. Phytochemical analysis and total antioxidant capacity of rhizome, above-ground vegetative parts and flower of three Iris species. Chem. Biodivers. 2019, 16, e1800565. [CrossRef] [PubMed]

132. Llorent-Martínez, E.J.; Córdova, M.L.F.; Zengin, G.; Bahadori, M.B.; Aumeeruddy, M.Z.; Rengasamy, K.R.R.; Mahomoodally, M.F. Parentucellia latifolia subsp. latifolia: A potential source for loganin iridoids by HPLC-ESI-MSn technique. J. Pharm. Biomed. Anal. 2019, 165, 374-380.

133. Llorent-Martínez, E.J.; Zengin, G.; Lobine, D.; Molina-García, L.; Mollica, A.; Mahomoodally, M.F. Phytochemical characterization, in vitro and in silico approaches for three Hypericum species. New J. Chem. 2018, 42, 5204-5214. [CrossRef]

134. Öztürk, G.; Ağalar, H.G.; Yildiz, G.; Göger, F.; Kirimer, N. Biological activities and luteolin derivatives of Verbascum eskisehirensis Karavel., Ocak Ekici. J. Res. Pharm. 2019, 23, 532-542. [CrossRef]

135. Bączek, K.B.; Wiśniewska, M.; Przybył, J.L.; Kosakowska, O.; Weglarz, Z. Arbuscular mycorrhizal fungi in chamomile (Matricaria recutita L.) organic cultivation. Ind. Crops Prod. 2019, 140, 111562. [CrossRef]

136. Gomes, A.F.; Almeida, M.P.; Leite, M.F.; Schwaiger, S.; Stuppner, H.; Halabalaki, M.; Amaral, J.G.; David, J.M. Seasonal variation in the chemical composition of two chemotypes of Lippia alba. Food Chem. 2019, 273, 186-193. [CrossRef] 
137. Han, A.-R.; Nam, B.; Kim, B.-R.; Lee, K.-C.; Song, B.-S.; Kim, S.H.; Kim, J.-B.; Jin, C.H. Phytochemical composition and antioxidant activities of two different color chrysanthemum flower teas. Molecules 2019, 24, 329. [CrossRef]

138. Arsenijević, J.; Drobac, M.; Šoštarić, I.; Ražić, S.; Milenković, M.; Couladis, M.; Maksimović, Z. Bioactivity of herbal tea of Hungarian thyme based on the composition of volatiles and polyphenolics. Ind. Crops Prod. 2016, 89, 14-20. [CrossRef]

139. Cheng, S.; Shiea, J. Advanced spectroscopic detectors for identification and quantification: Mass spectrometry. In Instrumental Thin-Layer Chromatography; Poole, C.F., Ed.; Elsevier Inc.: Amsterdam, The Netherlands, 2015; p. 251.

140. Shao, Y.; Wu, Q.; Wen, H.; Chai, C.; Shan, C.; Yue, W.; Yan, S.; Xu, H. Determination of flavones in Lophatherum gracile by liquid chromatography tandem mass spectrometry. Instrum. Sci. Technol. 2014, 42, 173-183. [CrossRef]

141. Sánchez-Rabaneda, F.; Jauregui, O.; Casals, I.; Andres-Lacueva, C.; Izquierdo-Pulido, M.; Lamuela-Raventós, R.M. Liquid chromatographic/electrospray ionization tandem mass spectrometric study of the phenolic composition of cocoa (Theobroma cacao). J. Mass Spectrom. 2003, 38, 35-42. [CrossRef] [PubMed]

142. Lin, L.-Z.; Harnly, J.M. Identification of the phenolic components of chrysanthemum flower (Chrysanthemum morifolium Ramat). Food Chem. 2010, 120, 319-326. [CrossRef]

143. Liu, R.; Yan, W.; Han, Q.; Lv, T.; Wang, X.; Liu, X.; Fan, X.; Meng, C.; Wang, C. Simultaneous detection of four flavonoids and two alkaloids in rat plasma by LC-MS/MS and its application to a comparative study of the pharmacokinetics between Abri Herba and Abri mollis Herba extract after oral administration. J. Sep. Sci. 2019, 42, 1341-1350. [CrossRef] [PubMed]

144. Ye, Y.; Mo, S.; Feng, W.; Ye, X.; Shu, X.; Long, Y.; Guan, Y.; Huang, J.; Wang, J. The ethanol extract of Involcucrum castaneae ameliorated ovalbumin-induced airway inflammation and smooth muscle thickening in guinea pigs. J. Ethnopharmacol. 2019, 230, 9-19. [CrossRef] [PubMed]

145. Escher, G.B.; Santos, J.S.; Rosso, N.D.; Marques, M.B.; Azevedo, L.; do Carmo, M.A.V.; Daguer, H.; Molognoni, L.; Prado-Silva, L.D.; Sant'Ana, A.S.; et al. Chemical study, antioxidant, anti-hypertensive, and cytotoxic/cytoprotective activities of Centaurea cyanus L. petals aqueous extract. Food Chem. Toxicol. 2018, 118, 439-453. [CrossRef]

146. Janković, T.; Zdunić, G.; Beara, I.; Balog, K.; Pljevljakušić, D.; Stešević, D.; Šavikin, K. Comparative study of some polyphenols in Plantago species. Biochem. Syst. Ecol. 2012, 42, 69-74. [CrossRef]

147. Pintać, D.; Četojević-Simin, D.; Berežni, S.; Orčić, D.; Mimica-Dukić, N.; Lesjak, M. Investigation of the chemical composition and biological activity of edible grapevine (Vitis vinifera L.) leaf varieties. Food Chem. 2019, 286, 686-695. [CrossRef]

148. Lin, L.-Z.; Lu, S.; Harnly, J.M. Detection and quantification of glycosylated flavonoid malonates in celery, Chinese celery, and celery seed by LC-DAD-ESI/MS. J. Agric. Food Chem. 2007, 55, 1321-1326. [CrossRef]

149. Siciliano, T.; De Tommasi, N.; Morelli, I.; Braca, A. Study of flavonoids of Sechium edule (Jacq) Swartz (Cucurbitaceae) different edible organs by liquid chromatography photodiode array mass spectrometry. J. Agric. Food Chem. 2004, 52, 6510-6515. [CrossRef]

150. İşcan, G.; Göger, F.; Demirci, B.; Köse, Y.B. Chemical composition and biological activity of Nepeta cilicica. Bangladesh J. Pharmacol. 2017, 12, 204-209. [CrossRef]

151. Choi, J.Y.; Desta, K.T.; Lee, S.J.; Kim, Y.-H.; Shin, S.C.; Kim, G.-S.; Lee, S.J.; Shim, J.-H.; Hacimüftüoğlu, A.; Abd El-Aty, A.M. LC-MS/MS profiling of polyphenol-enriched leaf, stem and root extracts of Korean Humulus japonicus Siebold \& Zucc and determination of their antioxidant effects. Biomed. Chromatogr. 2018, 32, e4171. [PubMed]

152. Haliloglu, Y.; Ozek, T.; Tekin, M.; Goger, F.; Baser, K.H.C.; Ozek, G. Phytochemicals, antioxidant, and antityrosinase activities of Achillea sivasica Çelik and Akpulat. Int. J. Food Prop. 2017, 20, S693-S706. [CrossRef]

153. Graça, V.C.; Dias, M.I.; Barros, L.; Calhelha, R.C.; Santos, P.F.; Ferreira, I.C.F.R. Fractionation of the more active extracts of Geranium molle L.: A relationship between their phenolic profile and biological activity. Food Funct. 2018, 9, 2032-2042. [CrossRef] [PubMed] 
154. Pereira, J.M.; Peixoto, V.; Teixeira, A.; Sousa, D.; Barros, L.; Ferreira, I.C.F.R.; Vasconcelos, M.H. Achillea millefolium L. hydroethanolic extract inhibits growth of human tumor cell lines by interfering with cell cycle and inducing apoptosis. Food Chem. Toxicol. 2018, 118, 635-644. [CrossRef]

155. Gonçalves, G.A.; Corrêa, R.C.G.; Barros, L.; Dias, M.I.; Calhelha, R.C.; Correa, V.G.; Bracht, A.; Peralta, R.M.; Ferreira, I.C.F.R. Effects of in vitro gastrointestinal digestion and colonic fermentation on a rosemary (Rosmarinus officinalis L) extract rich in rosmarinic acid. Food Chem. 2019, 271, 393-400. [CrossRef]

156. Soltana, H.; De Rosso, M.; Lazreg, H.; Vedova, A.D.; Hammami, M.; Flamini, R. LC-QTOF characterization of non-anthocyanic flavonoids in four Tunisian fig varieties. J. Mass Spectrom. 2018, 53, 817-823. [CrossRef]

157. Gaspar, M.C.; Fonseca, D.A.; Antunes, M.J.; Frigerio, C.; Gomes, N.G.M.; Vieira, M.; Santos, A.E.; Cruz, M.T.; Cotrim, M.D.; Campos, M.G. Polyphenolic characterisation and bioactivity of an Oxalis pes-caprae L. leaf extract. Nat. Prod. Res. 2018, 32, 732-738. [CrossRef]

158. Kite, G.C.; Porter, E.A.; Denison, F.C.; Grayer, R.J.; Veitch, N.C.; Butler, I.; Simmonds, M.S.J. Data-directed scan sequence for the general assignment of C-glycosylflavone O-glycosides in plant extracts by liquid chromatography-ion trap mass spectrometry. J. Chromatogr. A 2006, 1104, 123-131. [CrossRef]

159. Paulina Tambunan, A.; Bahtiar, A.; Tjandrawinata, R. Influence of extraction parameters on the yield, phytochemical, TLC-densitometric quantification of quercetin, and LC-MS profile, and how to standardize different batches for long term from Ageratum conyoides L. leaves. Pharmacogn. J. 2017, 9, 767-774. [CrossRef]

160. Mincsovics, E.; Ott, P.; Alberti, A.; Böszörményi, A.; Héthelyi, E.B.; Szoke, É.; Kery, A.; Lemberkovics, E.; Móricz, Á. In-situ clean-up and OPLC fractionation of chamomile flower extract to search active components by bioautography. JPC J. Planar Chromatogr. 2013, 26, 172-179. [CrossRef]

161. de Alves, G.A.D.; Oliveira de Souza, R.; Ghislain Rogez, H.L.; Masaki, H.; Fonseca, M.J.V. Cecropia obtusa extract and chlorogenic acid exhibit anti aging effect in human fibroblasts and keratinocytes cells exposed to UV radiation. PLoS ONE 2019, 14, e0216501. [CrossRef] [PubMed]

162. Ghouti, D.; Rached, W.; Abdallah, M.; Pires, T.C.S.P.; Calhelha, R.C.; Alves, M.J.; Abderrahmane, L.H.; Barros, L.; Ferreira, I.C.F.R. Phenolic profile and in vitro bioactive potential of Saharan Juniperus phoenicea L. and Cotula cinerea (Del) growing in Algeria. Food Funct. 2018, 9, 4664-4672. [CrossRef] [PubMed]

163. Venuprasad, M.P.; Kandikattu, H.K.; Razack, S.; Amruta, N.; Khanum, F. Chemical composition of Ocimum sanctum by LC-ESI-MS/MS analysis and its protective effects against smoke induced lung and neuronal tissue damage in rats. Biomed. Pharmacother. 2017, 91, 1-12. [CrossRef] [PubMed]

164. Majchrzak, T.; Wojnowski, W.; Lubinska-Szczygeł, M.; Różańska, A.; Namieśnik, J.; Dymerski, T. PTR-MS and GC-MS as complementary techniques for analysis of volatiles: A tutorial review. Anal. Chim. Acta 2018, 1035, 1-13. [CrossRef]

165. Nolvachai, Y.; Marriott, P.J. GC for flavonoids analysis: Past, current, and prospective trends. J. Sep. Sci. 2013, 36, 20-36. [CrossRef]

166. Zhang, K.; Zuo, Y. GC-MS determination of flavonoids and phenolic and benzoic acids in human plasma after consumption of cranberry juice. J. Agric. Food Chem. 2004, 52, 222-227. [CrossRef]

167. Creaser, C.S.; Koupai-Abyazani, M.R.; Stephenson, G.R. Capillary column gas chromatography of methyl and trimethylsilyl derivatives of some naturally occurring flavonoid aglycones and other phenolics. J. Chromatogr. A 1989, 478, 415-421. [CrossRef]

168. Schmidt, T.J.; Merfort, I.; Matthiesen, U. Resolution of complex mixtures of flavonoid aglycones by analysis of gas chromatographic-mass spectrometric data. J. Chromatogr. A 1993, 634, 350-355. [CrossRef]

169. Proestos, C.; Sereli, D.; Komaitis, M. Determination of phenolic compounds in aromatic plants by RP-HPLC and GC-MS. Food Chem. 2006, 95, 44-52. [CrossRef]

170. García-Villalba, R.; Pacchiarotta, T.; Carrasco-Pancorbo, A.; Segura-Carretero, A.; Fernández-Gutiérrez, A.; Deelder, A.M.; Mayboroda, O.A. Gas chromatography-atmospheric pressure chemical ionization-time of flight mass spectrometry for profiling of phenolic compounds in extra virgin olive oil. J. Chromatogr. A 2011, 1218, 959-971. [CrossRef]

171. Gao, X.; Williams, S.J.; Woodman, O.L.; Marriott, P.J. Comprehensive two-dimensional gas chromatography, retention indices and time-of-flight mass spectra of flavonoids and chalcones. J. Chromatogr. A 2010, 1217, 8317-8326. [CrossRef] [PubMed]

172. Krafczyk, N.; Glomb, M.A. Characterization of phenolic compounds in rooibos tea. J. Agric. Food Chem. 2008, 56, 3368-3376. [CrossRef] [PubMed] 
173. Peng, J.; Fan, G.; Hong, Z.; Chai, Y.; Wu, Y. Preparative separation of isovitexin and isoorientin from Patrinia villosa Juss by high-speed counter-current chromatography. J. Chromatogr. A 2005, 1074, 111-115. [CrossRef] [PubMed]

174. Waksmundzka-Hajnos, M.; Sherma, J.; Kowalska, T. Overview of the field of TLC in phytochemistry and the structure of the book. In Thin Layer Chromatography in Phytochemistry; Waksmundzka-Hajnos, M., Sherma, J., Kowalska, T., Eds.; CRC Press, Taylor \& Francis Group: Boca Raton, FL, USA, 2008; p. 5.

175. Skalicka-Woźniak, K.; Mroczek, T.; Kozioł, E. High-performance countercurrent chromatography separation of Peucedanum cervaria fruit extract for the isolation of rare coumarin derivatives. J. Sep. Sci. 2015, 38, 179-186. [CrossRef] [PubMed]

176. Sieniawska, E.; Skalicka-Woźniak, K. Isolation of chlorogenic acid from Mutellina purpurea L. herb using high-performance counter-current chromatography. Nat. Prod. Res. 2014, 28, 1936-1939. [CrossRef] [PubMed]

177. Wang, X.; Cheng, C.; Sun, Q.; Li, F.; Liu, J.; Zheng, C. Isolation and purification of four flavonoid constituents from the flowers of Paeonia suffruticosa by high-speed counter-current chromatography. J. Chromatogr. A 2005, 1075, 127-131. [CrossRef]

178. Leitão, S.G.; Leitão, G.G.; Vicco, D.K.T.; Pereira, J.P.B.; de Morais Simão, G.; Oliveira, D.R.; Celano, R.; Campone, L.; Piccinelli, A.L.; Rastrelli, L. Counter-current chromatography with off-line detection by ultra high performance liquid chromatography/high resolution mass spectrometry in the study of the phenolic profile of Lippia origanoides. J. Chromatogr. A 2017, 1520, 83-90. [CrossRef]

179. Fiamegos, Y.C.; Nanos, C.G.; Vervoort, J.; Stalikas, C.D. Analytical procedure for the in-vial derivatization-extraction of phenolic acids and flavonoids in methanolic and aqueous plant extracts followed by gas chromatography with mass-selective detection. J. Chromatogr. A 2004, 1041, 11-18. [CrossRef]

(C) 2019 by the authors. Licensee MDPI, Basel, Switzerland. This article is an open access article distributed under the terms and conditions of the Creative Commons Attribution (CC BY) license (http://creativecommons.org/licenses/by/4.0/). 